\title{
ESTADIOS DEL ISÓTOPO DE CARBONO: UN MODELO CRONOLÓGICO BASADO EN LAS VARIACIONES SECULARES DEL CARBONO-14 ATMOSFÉRICO
}

\author{
LUIS SALCEDO CAMACHO \\ PONTIFICIA UNIVERSIDAD CATÓLICA DEL PERÚ \\ Isalcedo@pucp.edu.pe
}

\begin{abstract}
RESUMEN
El presente artículo busca explicar los Estadios del Isótopo de Carbono o CIS (Salcedo 2011) a partir de las variaciones seculares del Carbono-14 atmosférico. Tal propuesta se basa en un fenómeno advertido por el autor en 1997, al calibrar series de fechados que resultaban en conglomerados generados por la 'topografía' de las curvas de calibración radiocarbónica. Los estadios temporales resultantes concuerdan con las variaciones cíclicas de mediano plazo en la actividad solar, registradas por diversas disciplinas científicas a mediados del siglo XX (De Vries 1958; Dewey 1960; Link 1955; Schove 1955; Suess 1961), donde los grandes mínimos solares marcan el final de cada estadío. Esta aproximación alternativa a la cronología es aplicable a la Arqueología así como otras disciplinas del Cuaternario, permitiendo contrastar la contemporaneidad regional e interregional en un contexto natural, en lugar de cultural, liberando al arqueólogo de las particularidades cronológicas de las tradicionales secuencias culturales locales.
\end{abstract}

Palabras Clave: Radiocarbono, Calibración radiocarbónica, Estadios del Isótopo de Carbono.

\begin{abstract}
The present article explains the Carbon Isotope Stages or CIS (Salcedo 2011) starting from the secular variations in the atmospheric Carbon-14. Such proposal is based on a phenomenon noticed by the author in 1997; during the calibration of a series of dates resulting in clusters generated by the 'topography' of the Radiocarbon calibration curves. The resulting temporal stages are in concordance with medium-term cyclic variations in the solar activity, recorded by different scientific disciplines in mid-XXth century (De Vries 1958; Dewey 1960; Link 1955; Schove 1955; Suess 1961), where the Grand Solar Minima mark the end of each stage. This alternative approach to chronology can be applied to Archaeology as well other Quaternary disciplines, allowing to contrast regional and interregional contemporaneity in a natural context, instead of cultural, liberating archaeologist from the chronological particularities of the classic local cultural sequences
\end{abstract}

KeYwords: Radiocarbon, Radiocarbon calibration, Carbon Isotope Stages.

i Doctor en arqueología por la Universidad de Varsovia, Polonia; licenciado y bachiller en arqueología por la Pontificia Universidad Católica del Perú. Profesor de la Pontificia Universidad Católica del Perú. Jefe de Proyectos Arqueológicos en Ecología y Tecnología Ambiental S.A. (Grupo Graña y Montero). 
«La armonía invisible

es más fuerte que la visible.»

(Heráclito de Efeso [n.535-m.482 BC]: Fragmento 54 [Trad. de Mónica Cavallé]).

«Podemos ver hacia delante en el estudio de las variaciones del clima y el Sol como una interacción fructífera entre arqueólogos y geofísicos, como aquella que se dio durante el desarrollo de la datación por 14C.»

(S. Keith Runcorn 1990: 686 [La traducción es mía]).

\section{INTRODUCCIÓN}

Durante más de siglo y medio, la determinación de la antigüedad de los contextos y artefactos desenterrados por los arqueólogos han sido materia de arduas y cuasi interminables discusiones. Tras superar la etapa de la datación por métodos relativos -aquellos aplicables a un sitios o contextos particulares-, gracias al aporte de las ciencias naturales, se llegó al desarrollo de diversos métodos de datación absoluta, siendo uno de los primeros y más usado aquel del radiocarbono $\left({ }^{14} \mathrm{C}\right)$, desarrollado por Libby y sus colegas de la Universidad de California en Berkeley, EUA, entre 1935 y 1949. Dicho método provee una escala de tiempo absoluto independiente para correlacionar eventos a una escala global (Vogel 2002: 159), llegando hasta los 60000 años (Blackwell \& Buck 2003: 3), revolucionando así los estudios prehistóricos cuaternarios en diversos campos del conocimiento humano (Anderson et al. 1947: 576; De Vries 1959: 169; Libby 1960: 609; ver también Berger 1983; Blackwell \& Buck 2003: 1; Damon, Lerman \& Long 1978: 473; Renfrew 1990: 260; Taylor 1985: 309).

Desde mediados del siglo XX, el método de datación por radiocarbono ha sido la herramienta fundamental de los arqueólogos a nivel mundial para el establecimiento de diversas cronologías. Sin embargo, apenas una década después de inventarse este método de datación se descubrieron las primeras incongruencias con el registro dendrocronológico (Broecker, Olson \& Bird 1959; Damon \& Long 1962; De Vries 1958, 1959; Münnich 1957; Ralph 1959; Ralph \& Stuckenrath 1960; Suess 1961), lo que ocasionó que poco después se publicaran las primeras curvas de calibración (Crowe 1958: 470 y Figura 1; Suess 1965: Tablas 2 y 3, Figura 4). A pesar de ello, muchos investigadores aún son reticentes a emplear la calibración radiocarbónica, y pocos han caído en la cuenta de cuán ilusorias pueden ser sus cronologías al haber sido éstas construidas a partir de fechas no calibradas (Salcedo 2006; Watkins 1975 [citado en Damon, Leman \& Long 1978: 475]).

El objetivo del presente artículo es explicar los Estadios del Isótopo de Carbono a partir de las variaciones seculares del Carbono-14 atmosférico. Sin embargo, por razones de espacio, prescindiremos del recuento de las nociones básicas y la problemática del método de datación absoluta por radiocarbono, así como de la calibración radiocarbónica, pues tales temas ya han sido tratados en el libro Tempus Solaris (Salcedo 2011). Alternativamente, el lector puede consultar los artículos de Velarde (1998) y León (2006), publicados previamente en esta misma revista, o bien los trabajos publicados en el extranjero, como los de Bowman (1990), Ziółkowski et al. (1994) y Bronk-Ramsey et al. (2006), por citar algunos.

\section{MitO DE LA CORRECCIÓN DE LOS FECHADOS RADIOCARBÓNICOS}

Desde mediados del siglo XX, la mayoría de arqueólogos y otros científicos sociales, desconocedores de los detalles técnicos del método inventado por Libby en 1947 (Anderson et al. 1947; Arnold \& Anderson 1957; Arnold \& Libby 1949; Libby 1946, 1960, 1963, 1967, 1973, 1980; Libby, Anderson \& Arnold 1949), pensaban que las fechas radiocarbónicas podían transformarse directamente en fechas calendáricas mediante un simple procedimiento denominado corrección, que consistía en sustraer el valor de 1950 a la media aritmética del fechado proporcionado por el laboratorio. Este rudimentario procedimiento refleja crudamente cuán burdos e incorrectos eran los conocimien- 
tos del grueso de la comunidad científica social acerca del método de Libby, más aún, tomando en cuenta que la totalidad de fuentes de variabilidad y error en los fechados de ${ }^{14} \mathrm{C}$ fueron descubiertas entre 1953 y 1959, mientras que la primera propuesta de curva de calibración fue publicada a fines de la década de 1950 (Crowe 1958). Este desconocimiento, e incluso entendimiento erróneo, del método de Libby ha creado gran confusión en la comunidad científica social, generando un sinnúmero de discusiones bizantinas acerca de cronologías basadas en las correcciones, las que además se hacían obviando el error estándar de las muestras, con lo que las simples correcciones se convertían en gruesas incorrecciones.

\section{VARIACIÓN DEL ${ }^{14} \mathrm{C}$ ATMOSFÉRICO}

Luego de ostentar el título de invicto por seis años, el método desarrollado por Libby comenzó a exponer sus fuentes de inexactitud. Entre 1953 y 1958, se descubrió seis efectos que ocasionaban discrepancias entre las fechas radiocarbónicas y las fechas calendáricas esperadas (ver Salcedo 2011: 100-102). Aunque no siempre es reconocido, fue en realidad Karl O. Münnich quien descubrió las variaciones seculares en el ${ }^{14} \mathrm{C}$, aunque no lo expresó de manera tajante (Münnich 1957: 195; Willis, Tauber \& Munich 1960: 3). Münnich (1957: 195 y Tabla 1) analizó varias muestras de madera de roble y abeto de los siglos XIV-XIX AD, encontrando variaciones de hasta $1,81 \%$ en la actividad del ${ }^{14} \mathrm{C}$, lo que no es nada despreciable dado que una variación de $\pm 1 \%$ implica una diferencia de \pm 80 años. Sin embargo, Münnich (1957: 195) dijo explícitamente que no intentaba dar una explicación al fenómeno en ese momento.

Entre 1957 y 1959, varios laboratorios alrededor del mundo comenzaron a notar también las discrepancias, entendiendo que una o más de las premisas del método de Libby estaban erradas y necesitaban algún tipo de corrección (ver citas en Willis, Tauber \& Munich 1960: 4). No obstante, en ese momento, para Münnich ya no le quedaba duda que las variaciones existían, tal como fue expresado en un gráfico (Willis, Tauber \& Munich 1960: Figura 1). Lamentablemente, ya era tarde para este científico alemán, pues el crédito del descubrimiento de las variaciones en la actividad del ${ }^{14} \mathrm{C}$ había sido concedido a un colega holandés, que notó lo mismo que Münnich al año siguiente, pero que si se animó a darle una explicación.

En 1958, Hessel De Vries, a partir de sus estudios con muestras de los siglos XVI-XIX AD, descubrió “[...] que la concentración de Radiocarbono en la atmósfera ha fluctuado considerablemente.", pero dejó bien en claro que el Efecto Industrial (ver Salcedo 2011: 100-101) no podía explicar las variaciones anteriores al año $1850 \mathrm{AD}$, dado que las emisiones (de $12 \mathrm{CO}_{2}$ ) por la quema de combustibles fósiles fueron escasas antes de esa fecha (De Vries 1958: 96). Empleando una analogía de circuito eléctrico, De Vries (1958: 98) explicó que un cambio de 1\% en el indicador de producción del ${ }^{14} \mathrm{C}$ en la atmósfera produciría una variación de $1 \%$ en la concentración del ${ }^{14} \mathrm{C}$, pero esto no se lograría inmediatamente, sino luego del tiempo necesario para que los reservorios alcancen un nuevo estado de equilibrio. De ese modo, se tiene que el aumento de un $1 \%$ en la producción del ${ }^{14} \mathrm{C}$ produciría un incremento de solo 0,086\% en la concentración del ${ }^{14} \mathrm{C}$ luego de 20 años (si es que dicho aumento en la producción es sostenido); por ende, el incremento del $2 \%$ observado en la concentración del ${ }^{14} \mathrm{C}$ implicaría un aumento súbito en la producción del ${ }^{14} \mathrm{C}$ de casi $25 \%$ (siendo exactos: $2 \% \div 0,086=23,26 \%$ ), algo que es imposible de aceptar dado lo que se conoce acerca de la constancia del flujo de rayos cósmicos, y dado que se sabe que el campo magnético terrestre varía a muy largo plazo (De Vries 1958: 99; Houtermans: 1966: 6, 10, 12; ver también Salcedo 2011: 47-48, 83-87, 126-129).

En un primer momento, De Vries (1958: 99) pensó que la explicación a las rápidas fluctuaciones en el ${ }^{14} \mathrm{C}$ se debían a una razón geoquímica, vale decir, a una alteración en el sistema de intercambio entre los distintos reservorios de carbono, sobre todo entre los reservorios de la capa mezclada del océano y el océano profundo (ver Salcedo 2011: 96-98). Siguiendo los planteamientos de Broecker sobre la Circulación Termohalina, De Vries (1958: 100) explicó que un descenso en la temperatura media 
anual provocaría una alteración del proceso de recirculación vertical en los océanos (normalmente, el agua superficial cálida es reemplazada por agua profunda fría, pero en esta situación el agua superficial enfriada ya no sería reemplazada por el agua profunda, más "vieja" y con menos ${ }^{14} \mathrm{C}$ ); de este modo, el nivel de actividad del ${ }^{14} \mathrm{C}$ atmosférico aumentaría debido a que el ${ }_{12} \mathrm{C}$ del agua profunda ya no participaría, temporalmente, del intercambio con la atmósfera. Esto llevó a De Vries (1958: 101) a decir que:

“[...] puede ocurrir que tres o incluso cinco o más muestras de diferente edad tengan al presente la misma actividad; esto podría significar que las mediciones radiocarbónicas no darán un edad bien definida". (La traducción es mía).

Un año más tarde, poco antes de su temprana muerte, De Vries (1959: 187-188) esbozó el impacto de las futuras curvas de calibración en las fechas calendáricas, anotando que:

"Partiendo de la actividad en el presente de una muestra desconocida, su actividad en el pasado puede ser representada por una curva que se eleva exponencialmente (1 por ciento cada 80 años, doblando su altura en una vida media). El punto de intersección con la línea horizontal que representa la actividad inicial (constante) da la edad de la muestra. Sin embargo, si la actividad inicial no es constante, [siendo] representada por una línea 'ondulante' cuando es trazada respecto al tiempo, la curva exponencial puede cortarla en uno, tres, o cinco puntos (etc.), dando cada uno una edad compatible con el resultado de la medición del Radiocarbono." (La traducción es mía).

Las variaciones del ${ }^{14} \mathrm{C}$ atmosférico fueron corroboradas por trabajos posteriores (p.e. Stuiver 1961, 1970; Stuiver \& Suess 1966; Suess 1961, 1965, 1978, 1980; ver también Neftel, Oeschger \& Suess 1981), llegándose a la conclusión de que era necesario calibrar los resultados (Damon, Lerman \& Long 1978: 474; ver también Blackwell \& Buck 2003: 3).

El químico y físico nuclear austriaco Hans E. Suess fue el primero en tomar en serio el fenómeno descubierto por De Vries $(1958,1959)$. En 1960, en una conferencia informal realizada en Highland Park, Illinois, EUA, Suess (1961: 90) indicó acerca de la datación por radiocarbono:

"El método de está basado en las siguientes premisas: (1) constancia del flujo de rayos cósmicos; (2) constancia de los varios reservorios de intercambio de Carbono. Se ha encontrado empíricamente que esos supuestos eran correctos dentro de ciertos límites de error de las mediciones, pero refinamientos en la técnica de determinación del ${ }^{14} \mathrm{C}$ ahora han mostrado la existencia de desviaciones que demuestran que uno $\mathrm{o}$ ambos supuestos [mencionados] arriba no son precisamente correctos." (La traducción es mía).

Sin embargo, Suess (1961: 91) recalcó que:

"[...] en este momento, no puede darse una explicación concluyente para la causa de las fluctuaciones temporales del ${ }^{14} \mathrm{C}$." (La traducción es mía).

Después, Suess comentó acerca de lo hallado en 1961 (Suess 1965: 5938):

"Los resultados mostraron concluyentemente que las variaciones del tipo observado por investigadores previos existían. La naturaleza de la dependencia temporal de la actividad de ${ }^{14} \mathrm{C}$ atmosférico revelada por esas mediciones fueron bastante inesperadas: parecía que los máximos que ocurrieron unos pocos siglos atrás fueron precedidos por un amplio mínimo en la actividad del ${ }^{14} \mathrm{C}$ durante el cuarto o quinto siglo A.D. Previo a esa época, la actividad mostraba una tendencia decreciente por al menos 3000 años [...]" (La traducción es mía). 
En 1959, Suess solo contaba con alrededor de treinta fechados; eso le bastó para darse cuenta de que las variaciones de mediano plazo (o wiggles como él las llamaba) de verdad existían, confirmando y superando las observaciones anteriores (Münnich 1957; De Vries 1958). Entre 1963 y 1964, Suess consiguió 130 muestras adicionales (Suess 1965: 5938), lo que le permitió elaborar su primera curva de calibración, publicada al año siguiente (Suess 1965: Figura 4)ํ. Años después Suess (1978: 1; cf. Stuiver \& Suess 1966: 538) comentó que:

"La base para la validez de esta calibración es el bien conocido hecho que, para todo propósito práctico, las muestras de madera que crecieron al mismo tiempo muestran el mismo contenido radiocarbónico. ${ }^{2}$ Sin embargo, lo inverso no siempre es verdad: Las muestras de madera que muestran el mismo contenido radiocarbónico no tienen necesariamente la misma edad, debido a los embobinamientos (o wiggles) y escalones de la curva." (La traducción es mía).

Los detractores de Suess minimizaban la importancia, o incluso negaban la existencia, de las variaciones de mediano y corto plazo, indicando que eran artefactos producidos ya sea por errores de laboratorio (Libby 1952 [citado en Suess 1986: 259]; ver también Damon, Lerman \& Long 1978: 484; Pearson et al. 1977 [citado en Clark 1979: 51]), o sea por errores estadísticos (Clark 1975: 251, 1979: 54), o bien aceptando solo variaciones de largo plazo relacionadas al geomagnetismo (Damon, Lerman \& Long 1978: 477-482; Wendland \& Donley 1971: 138). Esto llevó a Suess (1978: 1) a comentar que:

"Mediciones por otros laboratorios confirmaron la tendencia general de la curva, pero no han contribuido a nuestro conocimiento de las fluctuaciones más rápidas en una escala de tiempo de 100 años, los llamados wiggles de la curva de calibración." (La traducción es mía).

El químico estadounidense Paul E. Damon resume las posibles causas de las fluctuaciones del ${ }^{14} \mathrm{C}$ de la siguiente manera (Damon, Lerman \& Long 1978: Tabla 3):

i) Variaciones en la relación $Q$ de producción de ${ }^{14} \mathrm{C}$ atmosférico por los rayos cósmicos, modulado a su vez por la actividad solar y los cambios en el campo magnético terrestre (además de los efectos derivados de las pruebas con bombas atómicas, entre otros factores) (ver también Suess 1968: 146);

ii) Variaciones en la razón de intercambio de ${ }^{14} \mathrm{C}$ entre los varios reservorios geoquímicos y los cambios en el contenido relativo de $\mathrm{CO}_{2}$ de dichos reservorios (que incluyen la solubilidad del $\mathrm{CO}_{2}$, su disolución y "tiempos de residencia" según variaciones en la temperatura; los efectos de las variaciones en el nivel del mar sobre la circulación y capacidad marina; la asimilación de $\mathrm{CO}_{2}$ por parte de las biosferas terrestre y marina, y la relación de esta última con la salinidad y temperatura oceánica, disponibilidad de nutrientes, afloramiento de aguas profundas, etc.); $y$,

iii) Variaciones en la cantidad total de $\mathrm{CO}_{2}$ en la atmósfera, la biosfera y la hidrosfera (que incluyen cambios en el ratio de introducción de $\mathrm{CO}_{2}$ en la atmósfera vía vulcanismo y otros procesos de desgasificación de la litosfera; las variaciones en los reservorios sedimentarios que almacenan ${ }^{14} \mathrm{CO}_{2}$; y la liberación de $\mathrm{CO}_{2}$ antiguo, carente de ${ }^{14} \mathrm{C}$, mediante la combustión de combustibles fósiles por la actividad humana industrial y doméstica).

1 Una curva de calibración anterior fue publicada por Crowe (1958: 470 y Figura 1).

2 Esto es conocido como el Principio de Simultaneidad (ver Clark 1979: 48). 


\section{VARIACIONES CÍCLICAS DEL ${ }^{14}$ C ATMOSFÉRICO}

La actividad solar no es homogénea: cada baja en la actividad solar (evidenciada en una menor presencia de manchas solares y fortalecimiento del viento solar) conlleva a un aumento de la incidencia de rayos cósmicos en la atmósfera terrestre, y el consiguiente bombardeo de neutrones produce gran cantidad de isótopos de ${ }^{14} \mathrm{C}$ (Bray 1967: 640, 1971: 1243; Pazdur \& Pazdur 1994: 34; Sonett 1984: 250; Sonett \& Suess 1984: 142-143; Stuiver 1961: 273, 1965: 534, 1970: 454; Stuiver \& Braziunas 1989: 405; Stuiver \& Quay 1980: 11; Suess 1965: 5938, 1968).

Durante cada mínimo solar aumenta el ${ }^{14} \mathrm{C}$ atmosférico que queda atrapado en los anillos de crecimiento anual de los árboles, provocando que las muestras de madera correspondientes a esos eventos parezcan más jóvenes de lo que realmente son; tal desviación corresponde aproximadamente al 2\% de la norma (Eddy 1976: 1195). Este fenómeno, conocido como Efecto De Vries, se relaciona con las variaciones de mediano plazo observadas en el ${ }^{14} \mathrm{C}$ atmosférico (Münnich 1957; De Vries 1958, 1959; Suess 1961, 1965, 1968, 1978, 1980; ver también Neftel, Oeschger \& Suess 1981).

Estas variaciones, conocidas también como wiggles o ciclos de Suess, fueron descritas inicialmente por Suess (1965, 1967, 1968, 1970a,b, 1978, 1980, 1986; ver también Neftel, Oeschger \& Suess 1981: 146), continuador de los trabajos de De Vries tras su prematura muerte en 1959. Suess (1980: 201-202; 1986: 262) escribió que sus wiggles son variaciones justificables más allá del error estadístico experimental (contra Libby 1952 [citado en Suess 1986: 259]), ${ }^{3}$ independientes de la procedencia geográfica de las muestras, y constituyen rasgos típicos y periódicamente recurrentes en las curvas de calibración. Además, Suess (1980: 205) descubrió que el aumento en los valores del ${ }^{14} \mathrm{C}$ son más acelerados que los descensos, a razón de 20 y 40 años, respectivamente, para alcanzar casi un $1 \%$ de variación.

A pesar de cierto escepticismo inicial (Damon, Lerman \& Long 1978: 484), los wiggles de Suess fueron confirmados de manera independiente en la década de 1970 (De Jong, Mook \& Becker 1979: 49 y Figura 1; Stuiver 1970: 454-455). Sin embargo, no fueron ampliamente aceptados hasta mediados de la década de 1980, cuando otros laboratorios obtuvieron secuencias más largas y finas, empleando métodos de mayor resolución.

No obstante, y a pesar de los recortes presupuestarios derivados de las críticas de Libby (ver Suess 1978: 2, 1986: 260), Suess demostró definitivamente la existencia de sus wiggles mediante la comparación de dos secuencias fechadas por métodos diferentes: i) Conteo proporcional de gas (acetileno) aplicado en muestras de pino "cono en cerda" californiano; y, ii) Conteo por cintilación líquida aplicado en muestras de roble irlandés; ello demostró que la variación del ${ }^{14} \mathrm{C}$ era un fenómeno global, independiente de la latitud, altitud y continente de procedencia (Suess 1967: 143, 1970a: 307, 1979: 779; Suess \& Linick 1990: 407, 410).

Las variaciones cíclicas en la concentración del ${ }^{14} \mathrm{C}$ atmosférico se clasifican en tres categorías, según sus constantes de tiempo características: i) Largo plazo: ciclos multimilenarios; ii) Mediano plazo: ciclos centenarios a milenarios, y iii) Corto plazo: ciclos anuales a multidecadales (p.e. Pazdur \& Pazdur 1994: 33-34; Dergachev, Raspopov \& Vasiliev 2000: 489-490), y pueden describirse de la siguiente manera:

i) Variaciones de largo plazo (ciclo geomagnético: 11300 años; semiciclo de 5600 años): Son variaciones multimilenarias causadas por los cambios en la intensidad del momento dipolar del campo magnético terrestre ${ }^{4}$ donde la magnitud de desviación en la concentración de

3 Sonett \& Suess (1984: 142) afirman que las variaciones en el ${ }^{14} \mathrm{C}$ atmosférico, registradas en los anillos de crecimiento del pino "conos en cerda" (Pinus aristata), apuntan a que estas no son aleatorias y, por ende, no pueden ser causadas por errores experimentales. Sonett (1985: 383) cita fuentes adicionales que avalan el planteamiento anterior (p.e. una comparación inter-laboratorios publicada en 1982).

4 Dicha variación es de 8,75 $\pm 1,58 \times 1022 \mathrm{~A} \cdot \mathrm{m}^{2}$ (McElhinny \& Senanayake 1982: 45 y Tabla 2) (ver Salcedo 2011: Subcapítulo 4.3). 
${ }^{14} \mathrm{C}$ presenta una curva sinusoidal (Bucha 1969; Houtermans 1966; Suess 1970b, 1974, 1980; ver también Damon \& Linick 1986; Damon \& Sternberg 1989; Damon, Cheng \& Linick 1989; Damon, Lerman \& Long 1978; Sternberg \& Damon 1983);

ii) Variaciones de mediano plazo (wiggles o Ciclo de Suess del ${ }^{14} \mathrm{C}: 210 \pm 10$ años [equivalente al Ciclo de Schove de manchas solares]; Ciclo de Link: 400 \pm 100 años; Ciclo Hallstadtzeit: $2400 \pm 200$ años): También llamadas "variaciones seculares" (De Vries 1958: 99, 1959: 181, 187-188; Suess 1961: 90, 1965: 5938, 1970a: 303, 307 y 309, 1980: 205; Suess \& Linick 1990: 405) y Efecto De Vries (Damon \& Long 1962: 240), son variaciones centenarias del orden de 1-2\%, derivadas de la modulación que ejerce la actividad solar en el flujo de los rayos cósmicos, a su vez amplificada por los cambios climáticos derivados también de la actividad solar (Anderson 1992: 53; Beck et al. 2001: 2455; Sonett 1984: Tablas 1 y 2; Sonett \& Suess 1984: 141-142; Stuiver \& Braziunas 1989: 405-406; ver también Neftel et al. 1981; Roedle 1980 [ambos citados en Pazdur \& Pazdur 1994: 34]);

iii) Variaciones de corto plazo (Ciclo de Hellmann: 5,5 años; Ciclo de Schwabe: 11 $\pm 1,2$ años; Ciclo de Hale: 22 años; y Ciclo de Gleissberg: ca. 88 años): Variaciones menores, del orden de 0,5\%, asociadas a los ciclos decadales de actividad solar y, específicamente, con la aparición de las manchas solares, formando harmónicos cada 11 y 22 años, a su vez modulados a escala multidecadal en un ciclo de ca. 88 años (Hale 1908; Gleissberg 1939: 159, 1971: Tabla 1).

\section{Variaciones Seculares del ${ }^{14} \mathrm{C}$ y Estadios del Isótopo de Carbono (CIS)}

El "reloj isotópico" del ${ }^{14} \mathrm{C}$ no funciona de manera constante, pues cada vez que mengua la actividad solar (durante un mínimo solar) dicho reloj se detiene, formándose valles y mesetas (plateau) en la curva de calibración; pero cuando la actividad solar se reanuda (p.e. mayor frecuencia de manchas solares y reanudación del flujo del viento solar), el reloj isotópico comienza a funcionar nuevamente, creando así un "pico de recuperación" justo al final del plateau (Salcedo 2006: 136; ver también Eddy 1980: 742). Este pico de recuperación se presenta como una cresta abrupta, de longitud corta y amplitud relativamente alta, aunque a veces se presenta como una cresta redondeada, de longitud de onda larga, o como sucesión de dos o tres crestas abruptas de medianas proporciones (Fig. 1).

Los valles y mesetas observados en las distintas curvas de calibración constituyen un registro de los sucesivos grandes mínimos solares (Weiss 1990: 618; Wigley \& Nelly 1990: 549). Cabe notar que las variaciones de mediano y corto plazo del ${ }^{14} \mathrm{C}$ son muy sensibles a los cambios en la actividad solar, que a su vez promueven los cambios climáticos (Beck et al. 2001; Hughen et al. 2004: 202; Lohmann, Rimbu \& Dima 2004: 1045-1046).

A partir de la distribución de los principales wiggles, o variaciones seculares (de mediano plazo) en el ${ }^{14} \mathrm{C}$ atmosférico, puede establecerse una segmentación del tiempo en función de los ciclos seculares de decaimiento y recuperación del ${ }^{14} \mathrm{C}$, tomando como referencia justamente los picos de recuperación (Salcedo 2006: 136). De este modo, se plantea el establecimiento de 53 Estadios del Isótopo de Carbono o CIS (siglas en Inglés para Carbon Isotope Stages) ${ }^{5}$ que van desde el año 1950 AD hasta los 37800 años Cal. BC (Fig. 2). ${ }^{6}$

5 Inicialmente, estos estadios fueron denominados Periodos Radiocarbónicos Calibrados (Salcedo 1997, 1998, 2001) y luego Estadios Radiocarbónicos Calibrados. Sin embargo, en el presente trabajo se ha preferido emplear el término 'Estadios del Isótopo de Carbono', en concordancia con la terminología empleada en el caso del isótopo de oxígeno-18.

6 Aunque la actual curva de calibración, IntCal09 (Reimer et al. 2009), llega hasta los 48000 años Cal. BC, se considera que su nivel de precisión anterior a los 38000 años Cal. BC no permite de momento crear CIS adicionales. La curva anterior, IntCal04 (Reimer et al. 2004), solo permitió crear 39 CIS entre los 23100 años Cal. BC y los 1945 años Cal. AD (Salcedo 2006: Cuadro III.2). 
a)

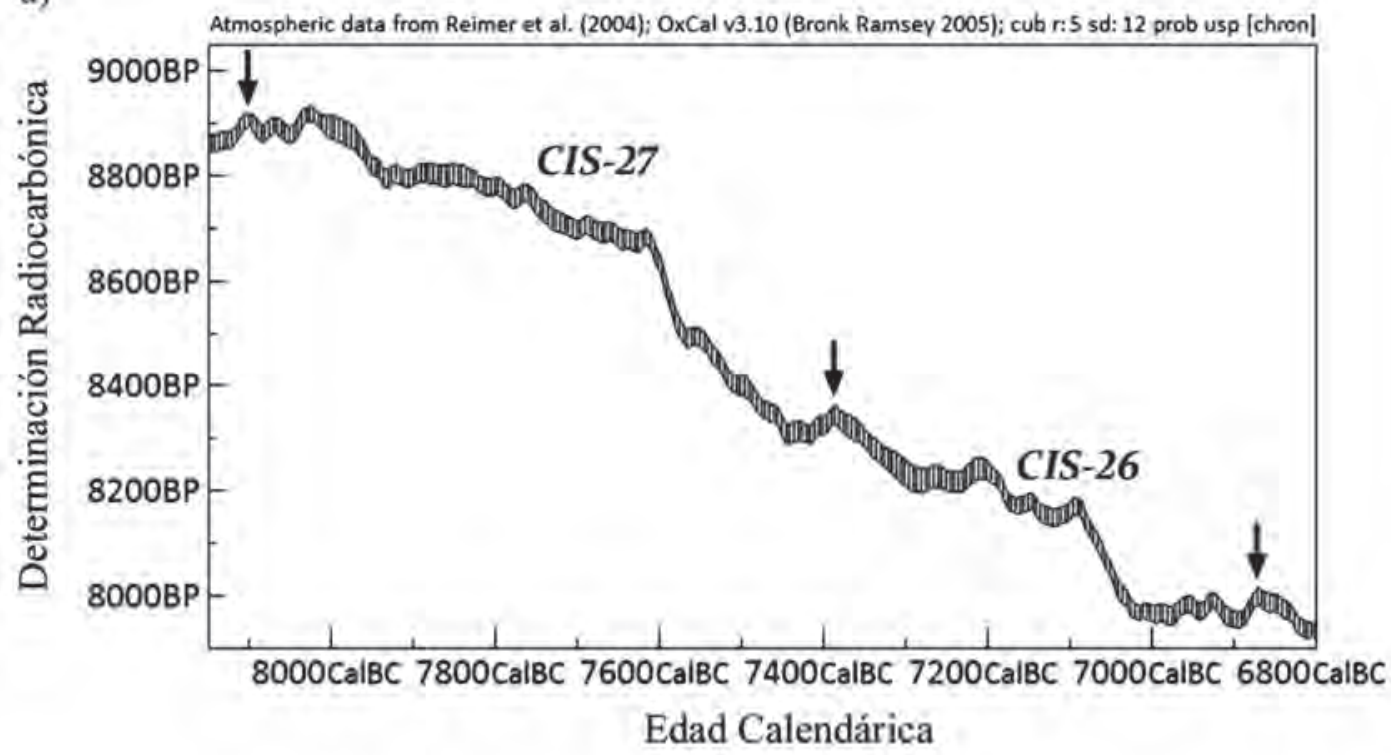

b)

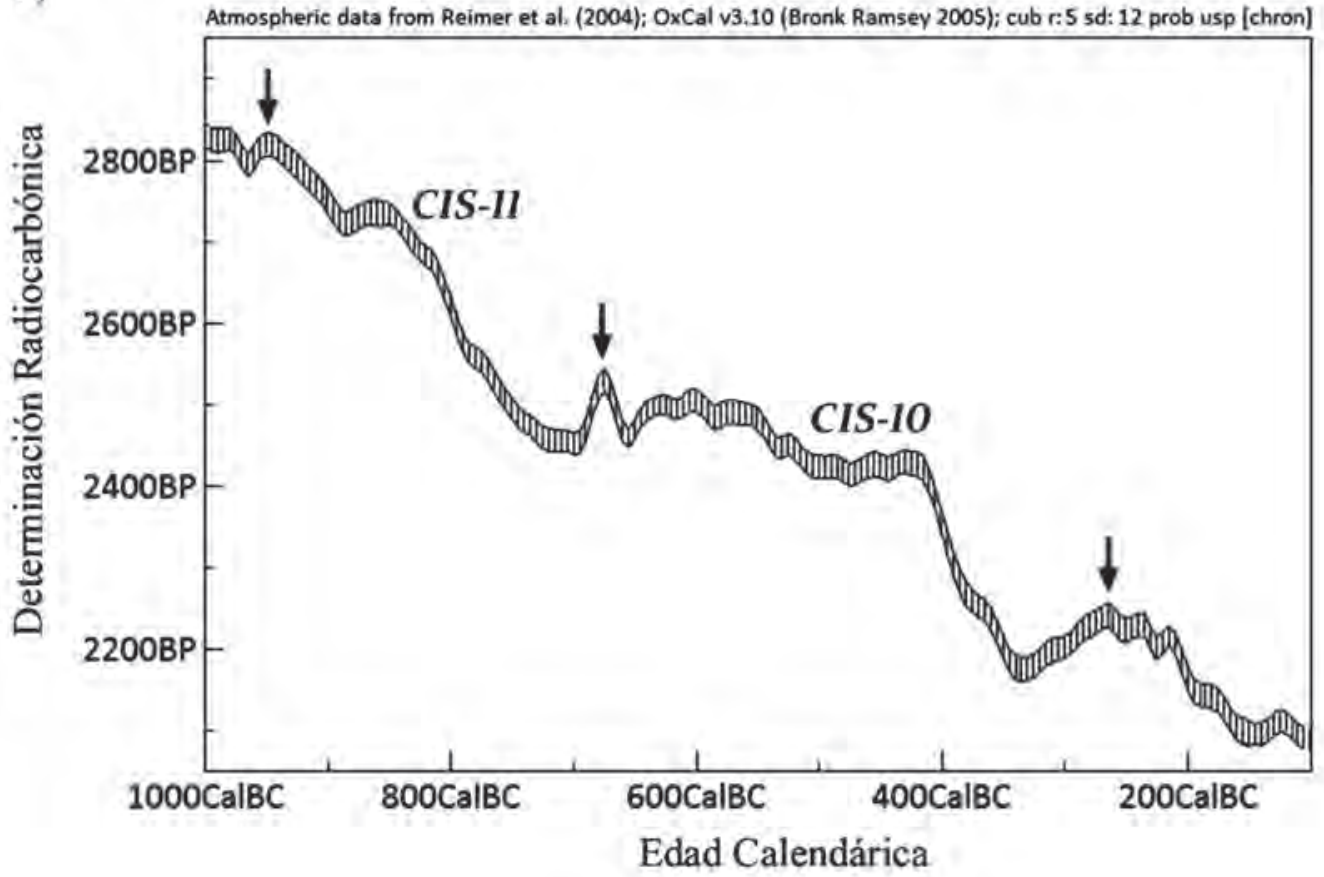

Figura 1. Estadios del Isótopo de Carbono (CIS): a) CIS-27 y 26; y, b) CIS-11 y 10. Los picos de recuperación al final de un valle o plateau se indican con una flecha (modificado a partir de Salcedo 2006: Tabla III.4 y Cuadro III.2; elaborado con el programa OxCal 3.10r [BronkRamsey 2005], a partir de la curva de calibración IntCal04 [Reimer et al. 2004]). Fuente: Programa OxCal v.3.10r disponible en: <http://www.rlaha.ox.ac.uk/orau/oxcal.html> 
Figura 2. Estadios del Isótopo de Carbono (CIS) según las curvas de calibración IntCalo9 (Reimer et al. 2009) para 41000-10000 años Cal. BC, e IntCal04 (Reimer et al. 2004) para 10000 años Cal. BC-1950 años Cal. AD. Se indica la numeración de cada CIS.
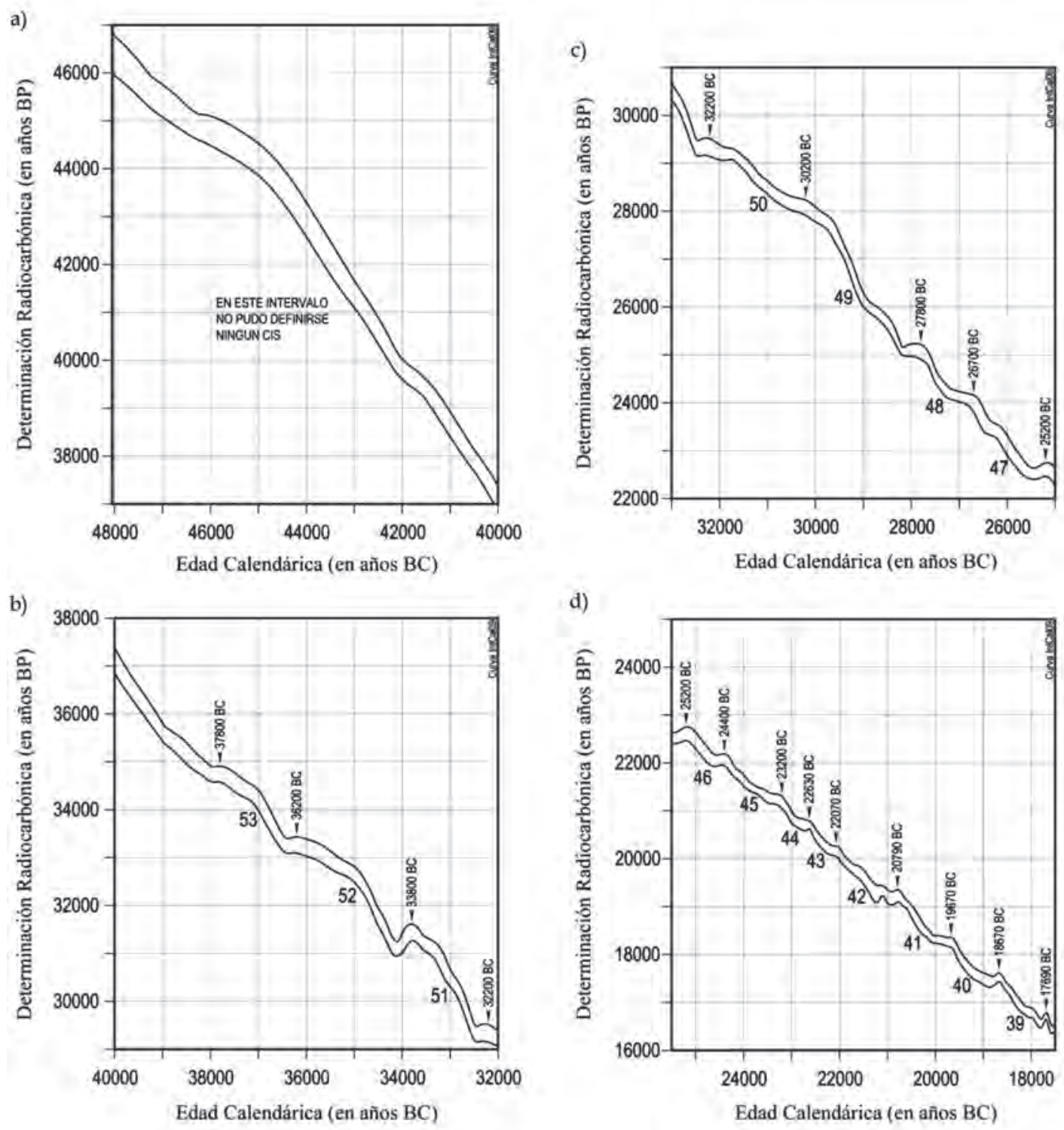

Continúa en la siguiente página 

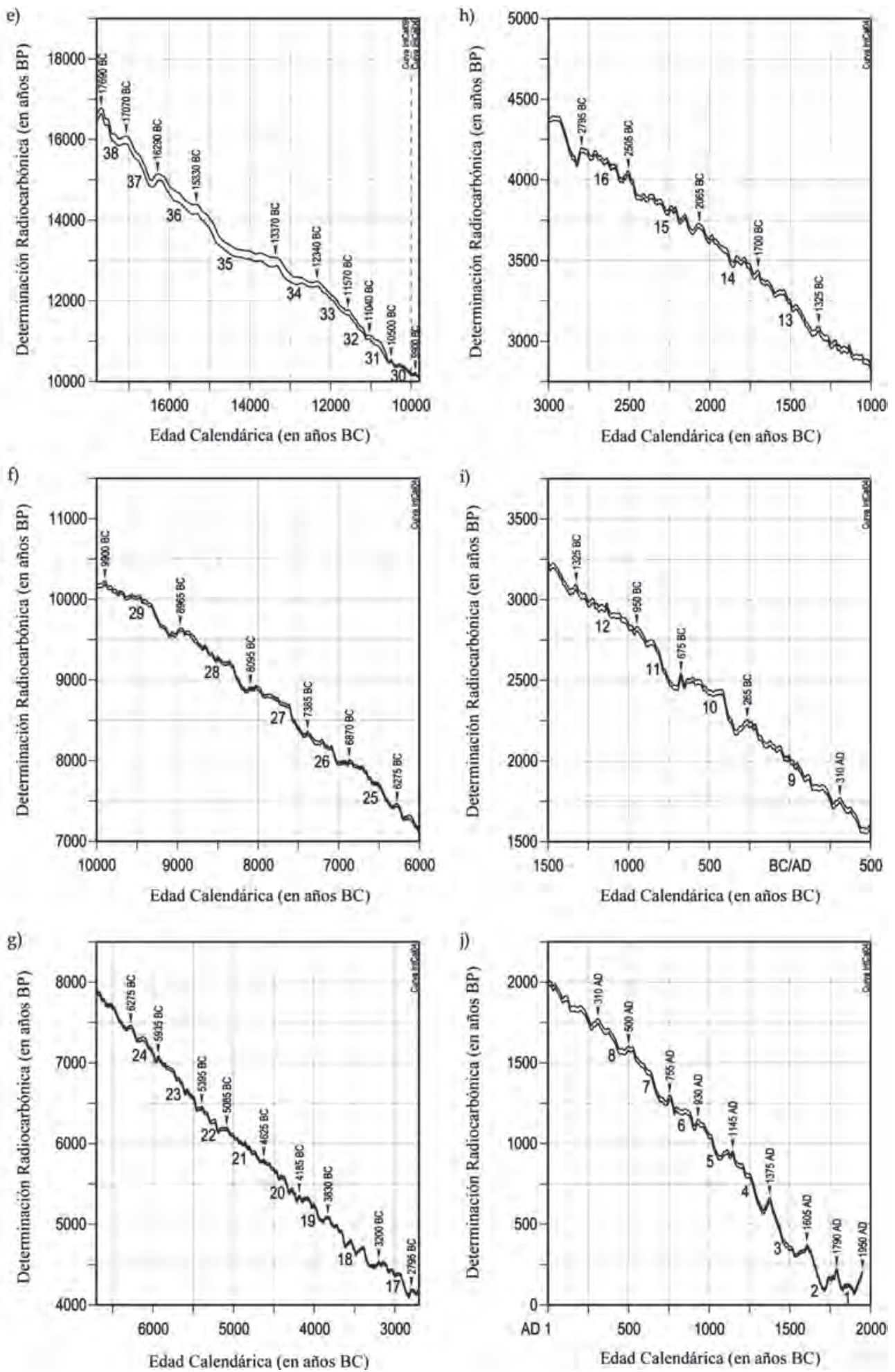
Figura 3. Estadios del Isótopo de Carbono (CIS) a partir de las variaciones seculares del ${ }^{14} \mathrm{C}$ atmosférico (cf. Salcedo 2006: Cuadro III.2): 37800-10000BC según curva IntCal09 (Reimer et al. 2009); 10000BC-1950AD según curva IntCal04 (Reimer et al. 2004).

\begin{tabular}{|c|c|c|c|c|c|c|c|c|}
\hline \multirow{2}{*}{$\begin{array}{l}\mathrm{CIS} \\
\left.{ }^{\prime \prime} \mathrm{C}\right)\end{array}$} & \multicolumn{3}{|c|}{ Cronologia abs. sin redondeo } & \multicolumn{2}{|c|}{ Cron. abs. con redondeo } & \multirow{2}{*}{$\begin{array}{l}\text { Estadios } \\
\text { Geológicos }\end{array}$} & \multirow{2}{*}{$\begin{array}{l}\text { Estadios Climáticos } \\
\text { (Zonas Climáticas) }\end{array}$} & \multirow{2}{*}{$\begin{array}{l}\mathrm{OIS} \\
\left({ }^{\prime 18} \mathrm{O}\right)\end{array}$} \\
\hline & Inicio & Fin & Difer. & Inicio & Fin & & & \\
\hline 0 & $1950 \mathrm{AD}$ & Hoy & $>60$ & $1950 \mathrm{AD}$ & Hoy & \multirow{14}{*}{ 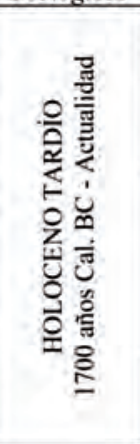 } & [Hipsitermal o Altitermal] & \multirow{35}{*}{1} \\
\hline 1 & $1790 \mathrm{AD}$ & $1950 \mathrm{AD}$ & 160 & $1800 \mathrm{AD}$ & $1950 \mathrm{AD}$ & & [Mesotermal] & \\
\hline 2 & $1605 \mathrm{AD}$ & $1790 \mathrm{AD}$ & 185 & $1600 \mathrm{AD}$ & $1800 \mathrm{AD}$ & & \multirow{3}{*}{$\begin{array}{l}\text { Pequeña Edad de Hielo } \\
\text { [Microtermal] }\end{array}$} & \\
\hline 3 & $1375 \mathrm{AD}$ & $1605 \mathrm{AD}$ & 230 & $1400 \mathrm{AD}$ & $1600 \mathrm{AD}$ & & & \\
\hline 4 & $1145 \mathrm{AD}$ & $1375 \mathrm{AD}$ & 230 & $1150 \mathrm{AD}$ & I400 AD & & & \\
\hline 5 & $930 \mathrm{AD}$ & $1145 \mathrm{AD}$ & 215 & $950 \mathrm{AD}$ & $1150 \mathrm{AD}$ & & \multirow{6}{*}{$\begin{array}{c}\text { Sub-Atlántico } \\
\text { [Hipsitermal o Altitermal] } \\
\text { Optimun climaticum } \\
\text { medieval }\end{array}$} & \\
\hline 6 & $755 \mathrm{AD}$ & $930 \mathrm{AD}$ & 175 & $750 \mathrm{AD}$ & $950 \mathrm{AD}$ & & & \\
\hline 7 & $500 \mathrm{AD}$ & $755 \mathrm{AD}$ & 255 & $500 \mathrm{AD}$ & $750 \mathrm{AD}$ & & & \\
\hline 8 & $310 \mathrm{AD}$ & $500 \mathrm{AD}$ & 190 & $300 \mathrm{AD}$ & $500 \mathrm{AD}$ & & & \\
\hline 9 & $265 \mathrm{BC}$ & $310 \mathrm{AD}$ & 575 & $250 \mathrm{BC}$ & $300 \mathrm{AD}$ & & & \\
\hline 10 & $675 \mathrm{BC}$ & $265 \mathrm{BC}$ & 410 & $700 \mathrm{BC}$ & $250 \mathrm{BC}$ & & & \\
\hline 11 & $950 \mathrm{BC}$ & $675 \mathrm{BC}$ & 275 & $950 \mathrm{BC}$ & $700 \mathrm{BC}$ & & \multirow{8}{*}{$\begin{array}{c}\text { Sub-Boreal } \\
\text { [Microtermal] }\end{array}$} & \\
\hline 12 & $1325 \mathrm{BC}$ & $950 \mathrm{BC}$ & 375 & $1300 \mathrm{BC}$ & $950 \mathrm{BC}$ & & & \\
\hline 13 & $1700 \mathrm{BC}$ & $1325 \mathrm{BC}$ & 375 & $1700 \mathrm{BC}$ & $1300 \mathrm{BC}$ & & & \\
\hline 14 & $2065 \mathrm{BC}$ & $1700 \mathrm{BC}$ & 365 & $2100 \mathrm{BC}$ & $1700 \mathrm{BC}$ & \multirow{10}{*}{ 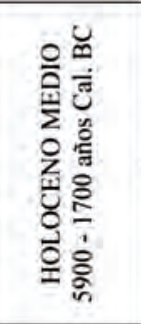 } & & \\
\hline 15 & $2505 \mathrm{BC}$ & $2065 \mathrm{BC}$ & 440 & $2500 \mathrm{BC}$ & $2100 \mathrm{BC}$ & & & \\
\hline 16 & $2795 \mathrm{BC}$ & $2505 \mathrm{BC}$ & 290 & $2800 \mathrm{BC}$ & $2500 \mathrm{BC}$ & & & \\
\hline 17 & $3200 \mathrm{BC}$ & $2795 \mathrm{BC}$ & 405 & $3200 \mathrm{BC}$ & $2800 \mathrm{BC}$ & & & \\
\hline 18 & $3830 \mathrm{BC}$ & $3200 \mathrm{BC}$ & 630 & $3800 \mathrm{BC}$ & $3200 \mathrm{BC}$ & & & \\
\hline 19 & $4185 \mathrm{BC}$ & $3830 \mathrm{BC}$ & 355 & $4200 \mathrm{BC}$ & $3800 \mathrm{BC}$ & & \multirow{5}{*}{$\begin{array}{c}\text { Atlántico Pleno } \\
\text { [Hipsitermal o Altitermal] } \\
\text { Optimun climaticum } \\
\text { neolitico }\end{array}$} & \\
\hline 20 & $4625 \mathrm{BC}$ & $4185 \mathrm{BC}$ & 440 & $4600 \mathrm{BC}$ & $4200 \mathrm{BC}$ & & & \\
\hline 21 & $5085 \mathrm{BC}$ & $4625 \mathrm{BC}$ & 460 & $5100 \mathrm{BC}$ & $4600 \mathrm{BC}$ & & & \\
\hline 22 & 5395 BC & $5085 \mathrm{BC}$ & 310 & $5400 \mathrm{BC}$ & $5100 \mathrm{BC}$ & & & \\
\hline 23 & $5935 \mathrm{BC}$ & $5395 \mathrm{BC}$ & 540 & $5900 \mathrm{BC}$ & $5400 \mathrm{BC}$ & & & \\
\hline 24 & $6275 \mathrm{BC}$ & $5935 \mathrm{BC}$ & 340 & $6300 \mathrm{BC}$ & $5900 \mathrm{BC}$ & \multirow{8}{*}{ 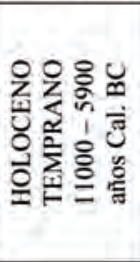 } & \multirow{4}{*}{$\begin{array}{l}\text { Atlántico Temprano } \\
\text { [Mesotermal] }\end{array}$} & \\
\hline 25 & $6870 \mathrm{BC}$ & $6275 \mathrm{BC}$ & 595 & $6900 \mathrm{BC}$ & $6300 \mathrm{BC}$ & & & \\
\hline 26 & $7385 \mathrm{BC}$ & $6870 \mathrm{BC}$ & 515 & $7400 \mathrm{BC}$ & $6900 \mathrm{BC}$ & & & \\
\hline 27 & 8095 BC & $7385 \mathrm{BC}$ & 710 & $8100 \mathrm{BC}$ & $7400 \mathrm{BC}$ & & & \\
\hline 28 & $8965 \mathrm{BC}$ & 8095 BC & 870 & $9000 \mathrm{BC}$ & $8100 \mathrm{BC}$ & & \multirow{4}{*}{$\begin{array}{l}\text { Pre-Boreal (PB) } \\
\text { [Microtermal] }\end{array}$} & \\
\hline 29 & $9900 \mathrm{BC}$ & $8965 \mathrm{BC}$ & 935 & $9900 \mathrm{BC}$ & $9000 \mathrm{BC}$ & & & \\
\hline 30 & $10500 \mathrm{BC}$ & $9900 \mathrm{BC}$ & 600 & $10500 \mathrm{BC}$ & $9900 \mathrm{BC}$ & & & \\
\hline 31 & $11040 \mathrm{BC}$ & $10500 \mathrm{BC}$ & 540 & $11000 \mathrm{BC}$ & $10500 \mathrm{BC}$ & & & \\
\hline 32 & $11570 \mathrm{BC}$ & $11040 \mathrm{BC}$ & 530 & $11600 \mathrm{BC}$ & $11000 \mathrm{BC}$ & \multirow{22}{*}{$\hat{\text { ê }}$} & & \\
\hline 33 & $12340 \mathrm{BC}$ & $11570 \mathrm{BC}$ & 770 & $12300 \mathrm{BC}$ & $11600 \mathrm{BC}$ & & 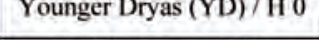 & \\
\hline 34 & $13370 \mathrm{BC}$ & $12340 \mathrm{BC}$ & 1030 & $13400 \mathrm{BC}$ & $12300 \mathrm{BC}$ & & Middle Dryas (MD) & \\
\hline 35 & $15330 \mathrm{BC}$ & $13370 \mathrm{BC}$ & 1960 & $15300 \mathrm{BC}$ & $13400 \mathrm{BC}$ & & Bolling / DO I & \\
\hline 36 & $16290 \mathrm{BC}$ & $15330 \mathrm{BC}$ & 960 & $16300 \mathrm{BC}$ & $15300 \mathrm{BC}$ & & Allerød & \\
\hline 37 & $17070 \mathrm{BC}$ & $16290 \mathrm{BC}$ & 780 & $17100 \mathrm{BC}$ & $16300 \mathrm{BC}$ & & & \\
\hline 38 & $17690 \mathrm{BC}$ & $17070 \mathrm{BC}$ & 620 & $17700 \mathrm{BC}$ & $17100 \mathrm{BC}$ & & Older Dryas (OD) / H I & \\
\hline 39 & $18670 \mathrm{BC}$ & $17690 \mathrm{BC}$ & 980 & $18700 \mathrm{BC}$ & $17700 \mathrm{BC}$ & & & \\
\hline 40 & $19670 \mathrm{BC}$ & $18670 \mathrm{BC}$ & 1000 & $19700 \mathrm{BC}$ & $18700 \mathrm{BC}$ & & a & \\
\hline 41 & $20790 \mathrm{BC}$ & $19670 \mathrm{BC}$ & 1120 & $20800 \mathrm{BC}$ & $19700 \mathrm{BC}$ & & b & \\
\hline 42 & $22070 \mathrm{BC}$ & $20790 \mathrm{BC}$ & 1280 & $22100 \mathrm{BC}$ & $20800 \mathrm{BC}$ & & & \\
\hline 43 & $22630 \mathrm{BC}$ & $22070 \mathrm{BC}$ & 560 & $22600 \mathrm{BC}$ & $22100 \mathrm{BC}$ & & c & 2 \\
\hline 44 & $23200 \mathrm{BC}$ & $22630 \mathrm{BC}$ & 570 & $23200 \mathrm{BC}$ & $22600 \mathrm{BC}$ & & & \\
\hline 45 & $24400 \mathrm{BC}$ & $23200 \mathrm{BC}$ & 1200 & $24400 \mathrm{BC}$ & $23200 \mathrm{BC}$ & & & \\
\hline 46 & $25200 \mathrm{BC}$ & $24400 \mathrm{BC}$ & 800 & $25200 \mathrm{BC}$ & $24400 \mathrm{BC}$ & & $\mathrm{DO}_{2}$ & \\
\hline 47 & $26700 \mathrm{BC}$ & $25200 \mathrm{BC}$ & 1500 & $26700 \mathrm{BC}$ & $25200 \mathrm{BC}$ & & Último Máximo Glacial & \\
\hline 48 & $27800 \mathrm{BC}$ & $26700 \mathrm{BC}$ & 1100 & $27800 \mathrm{BC}$ & $26700 \mathrm{BC}$ & & $(\mathrm{LGM}) / \mathrm{H} 2$ & \\
\hline 49 & $30200 \mathrm{BC}$ & $27800 \mathrm{BC}$ & 2400 & $30200 \mathrm{BC}$ & $27800 \mathrm{BC}$ & & DO 3-4+d-e & \\
\hline 50 & $32200 \mathrm{BC}$ & $30200 \mathrm{BC}$ & 2000 & $32200 \mathrm{BC}$ & $30200 \mathrm{BC}$ & & & \\
\hline 51 & $33800 \mathrm{BC}$ & $32200 \mathrm{BC}$ & 1600 & $33800 \mathrm{BC}$ & $32200 \mathrm{BC}$ & & H3 & \\
\hline 52 & $36200 \mathrm{BC}$ & $33800 \mathrm{BC}$ & 2400 & $36200 \mathrm{BC}$ & $33800 \mathrm{BC}$ & & $\mathrm{DO} 5+\mathrm{f}$ & \\
\hline 53 & $37800 \mathrm{BC}$ & $36200 \mathrm{BC}$ & 1600 & $37800 \mathrm{BC}$ & $36200 \mathrm{BC}$ & & $\mathrm{DO} 6+\mathrm{g}$ & 3 \\
\hline
\end{tabular}


Los CIS fueron definidos a partir de la ubicación de los respectivos picos de recuperación en las curvas de calibración IntCal09 (Reimer et al. 2009: para fechas entre 38000 y 10000 años Cal. BC) e IntCal04 (Reimer et al. 2004: para fechas entre 10000 años Cal. BC y 1950 años Cal. AD). ${ }^{7}$ Asimismo, para facilitar la recordación de los intervalos resultantes, se efectuó un redondeo por aproximación en múltiplos de cien años entre los 38000 y 1000 años Cal. BC, y en múltiplos de 50 años desde 1000 años Cal. BC en adelante (Fig. 3).

Aunque la presente propuesta está basada en los wiggles de Suess (o Efecto De Vries), no todos estos wiggles tienen la misma ponderación, debido a que presentan magnitudes diferentes, de manera que el efecto resultante es más bien una modulación del Ciclo de Suess (Suess 1965, 1980) produciendo periodos mayores como los del Ciclo de Link (Link 1955, 1961, 1963, 1977) y el Ciclo Hallstadtzeit (Houtermans 1971 [citado en Damon \& Linick 1986: 268-269]). La media general de los CIS es de $750 \pm 556,46$ años, aunque a continuación presentamos medias parciales, para ciertos intervalos arbitrarios de tiempo.

Considerando sus valores reales, sin redondeo, los CIS 1-8 (N=8; 15,1\%) abarcan el lapso 310-1950 $A D$, y su duración varía entre 160 y 255 años, con una media de $205 \pm 32,5$ años, lo que concuerda bastante bien con los valores conocidos del Ciclo de Suess. Los CIS 9-24 (N=16; 30,2\%) abarcan el lapso 6275 BC-310 AD, y su duración varía entre 275 y 540 años, con una media de 417,9 $\pm 100,6$ años, lo que constituye una modulación del Ciclo de Suess, también llamada Ciclo de Link. Los CIS 25-48 (N=24; 45,3\%) abarcan el lapso 27800-6275 BC, siendo su periodicidad algo más compleja, pues veintidós de ellos varían entre 515 y 1280, con una media de 821,1 \pm 239,3 años (la media y la desviación estándar son múltiplos del Ciclo de Suess en 4 y 1, respectivamente). Sin embargo, dos de ellos (los CIS 35 y 47) son modulaciones mayores, de 1500 y 1960 años, respectivamente, estando la última de ellas relacionada con el Ciclo Hallstadtzeit. Si consideramos estos tres valores mayores en la media anterior, ésta aumenta a 896,9 + 350,4 años. Finalmente, los últimos cinco intervalos, los CIS 49-53 (N=5; 9,4\%) abarcan el lapso 37800-27800 BC, y su duración varía entre 1600 y 2400 años, siendo también modulaciones del Ciclo Hallstadtzeit (Fig. 4).

Los ciclos detectados en la curva de calibración no son estáticos, sino que cambian de fase cada cierto tiempo. Esta modulación de fase fue notada por otros autores en diferentes campos de investigación:

i) En las secuencias dendrocronológicas (Douglass 1943: 24);

ii) En los cambios eustáticos en el nivel del mar (Mörner \& Rickard 1974: 145, 147, 150-151);

iii) En los ciclos de las manchas solares (Schove 1983: 393); $y$,

iv) En la variación del ${ }^{14} \mathrm{C}$ atmosférico (Sonett 1984: 248). Mientras Douglass (1943: 24) empleó el término inglés cyclic, Mörner (Mörner \& Rickard 1974: 150) empleó el término "ciclo irregular", para referirse a ciclos no estáticos y que cambian de periodicidad con el tiempo.

Los diferentes cambios de fase detectados en las curvas de calibración IntCal04 e IntCal09 son los siguientes:

i) En el periodo 37800-27800 BC (CIS 53-49) predomina el Ciclo Hallstadtzeit (H), ya sea el ciclo completo: 1H (2400 años), o una fracción de este: 2/3H (1600 años);

ii) En el periodo 27800-6275 BC (CIS 48-25) predomina la fracción 1/3H (800 años), aunque tam-

7 He mantenido los CIS originales (Salcedo 2006: Cuadro III.2), definidos a partir de la curva IntCal04 entre 12300 años Cal. BC y 1950 años Cal. AD (incluso aquellos de 12500-10000 años Cal. BC, dada la similitud entre ambas curvas en ese lapso). Varios de los CIS definidos previamente para 23100-12300 años Cal. BC fueron replanteados, dada la mayor resolución de la curva IntCal09, generándose otros nuevos para 23100-37800 años Cal. BC. 
bién aparecen las fracciones 1/2H (1200 años) y 1/5H (500 años);

iii) En el periodo 6275 BC-310 AD (CIS 24-9) predomina la fracción 1/6H (400 años =Ciclo de Link), con participación de las fracciones $1 / 4 H$ (600 años) y 1/8H (300 años); $y$,

iv) Finalmente, durante el periodo 310-1950 AD (CIS 8-1) predomina la fracción 1/12H (200 años = Ciclo de Suess), aunque también aparecen las fracciones 1/9H (250 años) y 1/15H (160 años) (ver también Salcedo 2011: Tabla 4).

Una posible explicación a los cambios de fase, en lo que respecta a la duración de las periodicidades observadas, quizás tenga que ver con factores heliomagnéticos (cambios internos propios del sol), o derivados del influjo de las mareas gravitacionales de los grandes planetas exteriores, como júpiter y saturno (Charvátová 1998 [citado en Charvátová 2000: 401]), que interactúan para "ordenar" y "desordenar" cíclicamente el movimiento inercial solar (Charvátová 2000: 400).

Las variaciones en la amplitud (intensidad) de las fluctuaciones del ${ }^{14} \mathrm{C}$ atmosférico parecen derivar del 'filtro' que impone la variación sinusoidal de largo plazo observada en el campo magnético terrestre, lo que provoca que las fluctuaciones del ${ }^{14} \mathrm{C}$ atmosférico sean mayores cuando el momento geomagnético es bajo, y sean menores cuando el momento geomagnético está alto (Suess 1970b: 599 y Figura 1; ver también Damon, Lerman \& Long 1978: Figura 6). Esto último concuerda con el patrón de amplitud observado, dado que entre fines del Pleistoceno Tardío y fines del Holoceno Temprano, 37800-6275 BC (CIS 53-25), predominan amplitudes de 2400 y 1200 años, mientras que entre fines del Holoceno Temprano e inicios del Holoceno Tardío, 6275 BC-310 AD (CIS 24-9), predominan amplitudes de 800, 600 y 400 años, quedando las amplitudes de 200 años confinadas a mediados y fines del Holoceno Tardío, 310-1950 AD.

Debe tenerse presente que las evidencias paleomagnéticas publicadas recientemente (Muscheler et al. 2005: Figuras 2, 5 y 7) muestran que el momento geomagnético, aunque oscilante, fue más débil durante el periodo comprendido entre 37800 y 6275 BC, lo que significa una mayor amplitud y variabilidad de la relación $\mathrm{Q}$ de producción del ${ }^{14} \mathrm{C}$ atmosférico, mientras que el pico de actividad geomagnética registrado hacia 310 AD explicaría la menor amplitud y variabilidad del ratio $Q$ registrada en el Holoceno Tardío respecto de las épocas anteriores, quedando el lapso medio ( 6275 BC-310 AD) en una

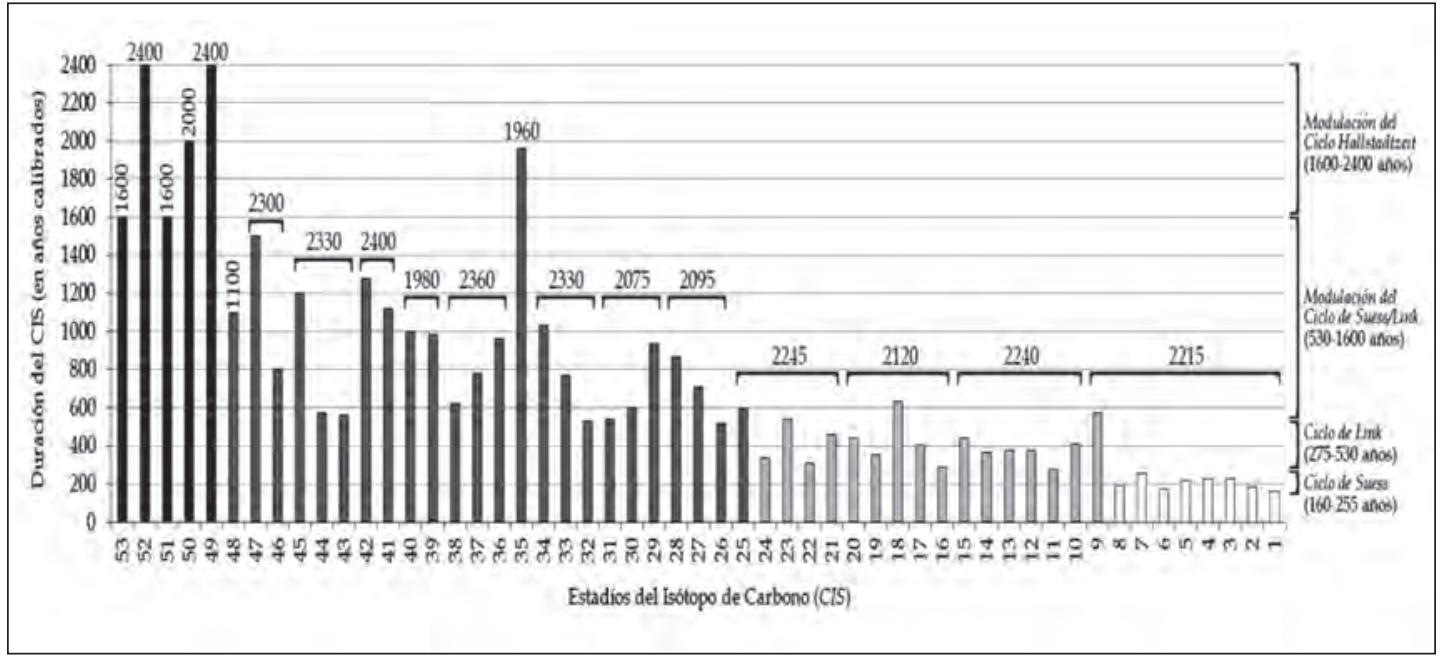

Figura 4. Variación en la duración de los Estadios del Isótopo de Carbono (CIS), mostrando las modulaciones del Ciclo Hallstadtzeit de 2400 años (en negro), el ciclo de 800 años (en gris oscuro), el Ciclo de Link de 400 años (en gris claro) y el Ciclo de Suess de 200 años. 
situación intermedia, aunque más cercano a la situación post- 310 AD (ver Salcedo 2011: Figuras 10c y 16), debido a que -como ya se dijo-, la actividad geomagnética general del Holoceno fue mayor a la del Pleistoceno Tardío (ver Salcedo 2011: Figura 11).

\section{IMPLICACIONES DEL MODELO}

Al usarse calibraciones con $2 \sigma(p=95,4 \%)$ se obtiene un considerable traslape entre los diferentes fechados de un mismo sitio, los que tienden a formar conglomerados dentro de los CIS, hecho que fue notado por el autor hace más de una década mientras desarrollaba su tesis de Licenciatura sobre el estadio Arcaico de los Andes Centrales (Salcedo 1997, 1998, 2001). En esa ocasión se empleó la curva de calibración Cal93 (Kromer \& Becker 1993; Stuiver \& Becker 1993; Stuiver \& Pearson 1993) aplicándola a 180 fechados y reconociendo los primeros CIS. Luego, el autor aplicó esta división cronológica en su tesis doctoral, sobre la prehistoria temprana de Sudamérica, empleando primero la curva IntCal98 (Stuiver et al. 1998) y luego la curva IntCal04 (Reimer et al. 2004) a una base de datos con más de 1850 fechados, los mismos que se agruparon siguiendo los CIS antes mencionados (Salcedo 2006).

Inicialmente, la agrupación de fechados calibrados de un complejo cultural dentro de un CIS fue interpretada como un mero 'artefacto' creado por las características propias de la curva de calibración (Salcedo 2006: 136), influenciados por la 'topografía' de la curva empleada. Este "defecto de origen" del método de radiocarbono presentaría dos consecuencias: una negativa y otra positiva:

La consecuencia negativa sería para los arqueólogos, historiadores, etc., pues los resultados de la calibración de fechados (ya sean individuales o en secuencias largas) hace difícil establecer cronologías de fases culturales finas (a nivel de décadas o fracciones de siglos), debido a la topografía de la curva de calibración y los errores estándares de las muestras (generalmente arriba de \pm 40 años con métodos convencionales), ${ }^{8}$ que tienden a 'oscurecer' periodicidades de corto plazo (p.e. Ciclo de Schwabe, Ciclo de Hale y Ciclo de Gleissberg, con una duración promedio de 11, 22 y 88 años, respectivamente).

Esto contradice las secuencias cronológicas empleadas actualmente, donde abundan las fases al interior de un mismo periodo cultural, lo que no puede sustentarse a nivel radiocarbónico calibrado debido al citado proceso de agrupación 'natural' de los fechados calibrados (Salcedo 2006: 137), aparentemente, según la serie de grandes mínimos solares.

Con el paso del tiempo crece la dependencia humana a la tecnología, lo que provoca una disminución de la capacidad de readaptación a situaciones ambientales y socioeconómicas derivadas de grandes cambios climáticos. Esto se expresa en el hecho de que las culturas precerámicas (mayormente referidas a poblaciones nómadas) parecen subsistir durante varios CIS contiguos (ver Salcedo 1997, 1998, 2006), presentando pocos cambios culturales en periodos relativamente largos, mientras que las culturas con cerámica (generalmente sedentarias), en especial durante los últimos 3000 años, comienzan a restringir su existencia a solo uno o dos CIS contiguos.

Algo que podría explicar este fenómeno, en parte, es el hecho de que el inicio de los grandes mínimos solares es abrupto, en comparación con su término más gradual (De Jong, Mook \& Becker 1979: 49; Stuiver 1980: Figura 1; Frick et al. 1997: 670, 678; Usoskin, Sokoloff \& Moss 2009: 349, 353).

Sin embargo, la consecuencia positiva sería para los geofísicos y paleoclimatólogos, pues significa la apertura a una serie de posibilidades para tratar de relacionar los cambios observados en la actividad solar con el clima terrestre, actual y pasado.

8 Nótese que incluso con un $\sigma= \pm 15$ años, típico del Método AMS, el intervalo de fechas radiocarbónicas a contrastar con la curva de calibración (usando $2 \sigma$ ) se amplia a 60 años ( \pm 30 años), lo que produce un intervalo de fechas calendáricas de alrededor de 50-80 años, o incluso mayor, dependiendo de la topografía del segmento correspondiente de la curva. 


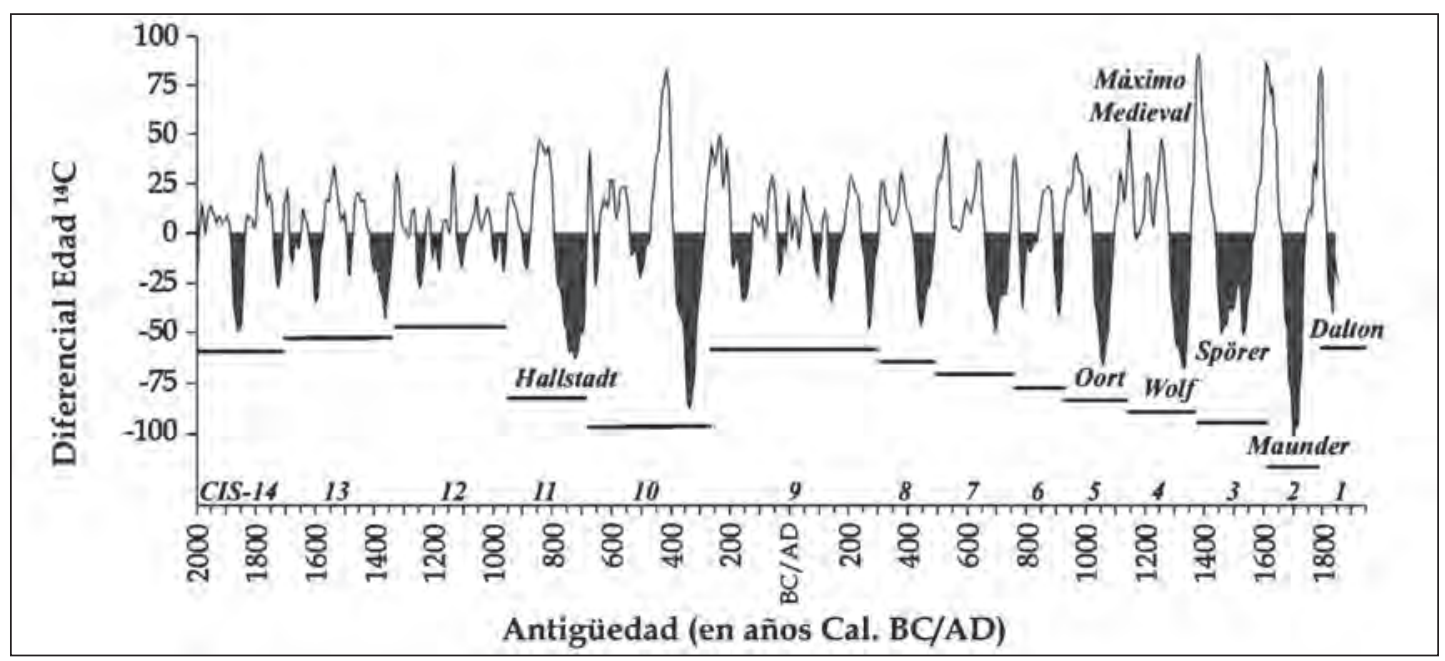

Figura 5. Detalle de la variación secular del ${ }^{14} \mathrm{C}$ para el periodo $2000 \mathrm{BC}-1850 \mathrm{AD}$ según la Curva IntCal04 <http://www.radiocarbon.org/IntCal04.htm>. Los grandes mínimos solares estan resaltados en color gris. Se indica la duración de cada CIS con barras negras y su numeración respectiva al pie de la figura.

Las evidencias discutidas en el presente trabajo sugieren que las grandes fluctuaciones observadas en las curvas de calibración -los wiggles de Suess o Efecto De Vries-, tienen en realidad implicancias de orden climático, toda vez que, como ya se explicó anteriormente, estos wiggles son un reflejo de la variabilidad del flujo de rayos cósmicos sobre la atmósfera superior terrestre (que dan origen al ${ }^{14} \mathrm{C}$ ), la misma que es modulada a su vez por la oscilación en la actividad solar, expresada en la frecuencia de manchas solares, auroras polares, etc. (p.e. Houtermans, Suess \& Monk 1967 [citado en Suess 1967: 599]; Stuiver 1961). Por ende, no es de sorprender que los wiggles (Ciclo de Suess de 200 años) y sus modulaciones, detectados en la calibración dendrocronológica del ${ }^{14} \mathrm{C}$, hayan sido también detectados en la frecuencia de manchas solares (Ciclo de Schove de 200 años) y en la frecuencia de las auroras polares (Ciclo de Link de 400 años) (Suess 1970b: 599).

Es clara la relación entre las variaciones de mediano plazo y los grandes mínimos solares (Salcedo 2011: 45-47), aunque algunos de los CIS abarcan más de uno de estos mínimos consecutivos, dado que están separados por tenues máximos (Fig. 5). En términos generales, los ciclos hallados en el presente estudio son concordantes con los resultados publicados por otros autores en las últimas décadas (p.e. Stuiver 1980, Stuiver \& Braziunas 1989; Stuiver \& Quay 1980; Usoskin 2008; Usoskin, Solanki \& Kovaltsov 2007). Algunas investigaciones han tratado de definir cuántos de estos eventos ocurrieron en los últimos miles de años, y si estos fueron de similares características en cuanto su amplitud (intensidad) y periodo (duración). Hace tres décadas, Stuiver (1980: 869) planteó que durante el Holoceno ocurrieron hasta 40 grandes mínimos solares que, según su duración, podrían clasificarse en dos tipos: i) Aquellos del tipo del Mínimo de Spörer, con una duración de alrededor de 240 años; y, ii) Aquellos del tipo del Mínimo de Maunder, más frecuentes, con una duración de 120-140 años. Dichos mínimos no serían variaciones aleatorias (es decir, al azar), sino que presentarían cierto patrón recurrente (Stuiver 1980: 869 y Figura 1). Tales conclusiones fueron corroboradas posteriormente, aunque fijándose lapsos más cortos, de 120-180 y 40-60 años, respectivamente (Stuiver \& Braziunas 1989: 406; Stuiver \& Quay 1980: 18). Recientemente, Usoskin y sus colegas confirmaron la existencia de 27 grandes mínimos solares para el periodo 9500 BC - 2000 AD (Usoskin, Solanki \& Kovaltsov 2007: 303-304, Tablas 1 y 2; ver también Usoskin 2008: 51-53). 


\section{CAMBio de PARAdigma}

Siempre ha sido un anhelo de los arqueólogos llegar a tener fechados realmente "absolutos", es decir, que las determinaciones radiocarbónicas hicieran referencia a fechas puntuales en lugar de los indeseables intervalos, que tantos dolores de cabeza y acaloradas discusiones han provocado en el último medio siglo. Un comentario de A. Colin Renfrew (1990: 659) resume muy bien este anhelo:

"[...] hay al menos la esperanza de que, para algunos rangos de tiempo, eventos marcadores mundiales puedan un día ser usados para permitir que ciertos fechados sean establecidos dentro de un solo año. [...]. Existe el prospecto, por ende, de usar esas correlaciones entre disciplinas para ofrecer una precisión cronológica dentro de un solo año (en lugar de la precisión restringida a varias décadas ofrecida por la determinación radiocarbónica directa), posiblemente aplicable en varias partes del mundo." (La traducción es mía).

El deseo de Renfrew se ve reflejado en curvas de calibración como la publicada por su asistente, R. Malcolm Clark (1975: Figura 1, 1979: Figura 1), que consiste en una curva 'suavizada' que prácticamente trata de ocultar la existencia de los wiggles, hecho que concuerda con la propuesta de Switsur (1973 [citado en Clark 1975: Figura 2]) y el pensamiento inicial de Paul E. Damon, quien hasta 1978 seguía afirmando que los wiggles “[...] aún no han sido confirmados." (Damon, Lerman \& Long 1978: 484).

Las características propias de las curvas de calibración (causadas por el Efecto De Vries o los wiggles de Suess) podrían ser aprovechadas para crear intervalos temporales estándares que faciliten la comparación interregional en Arqueología y en otras ciencias a nivel global (ver Salcedo 2006). En ese sentido, Bernd Kromer, de la Academia de Ciencias de Heidelberg, escribió recientemente (Kromer 2009: 17):

"Generalmente[,] las fluctuaciones atmosféricas del ${ }^{14} \mathrm{C}$ limitan la precisión de las edades radiocarbónicas calibradas, debido a las múltiples coincidencias de las edades radiocarbónicas con la curva de calibración. Sin embargo, las fluctuaciones ('wiggles' de la curva) pueden ser tornadas para un buen uso si una secuencia ordenada de fechados ${ }^{14} \mathrm{C}$ es medida." (La traducción es mía).

Esto podría ser percibido como una limitación por aquellos colegas que persiguen la calibración a escala decadal (con ayuda del AMS) a fin de sustentar sus fases culturales, lo que el arqueólogo inglés Trevor Watkins denominó "el sueño de que los fechados ${ }^{14} \mathrm{C}$ eran absolutos" (Watkins 1975 [citado en Damon, Lerman \& Long 1978: 475; Damon Long \& Lerman 1978: 329]). El 'anhelo' del fechado 'puntual' fue calificado hace unos años como "el nuevo Santo Grial de la Arqueología" (Salcedo 2006: 137). En ese sentido, Suess escribió apropiadamente (Suess \& Linick 1990: 407):

"Por muchas razones, los arqueólogos no deberían esperar que los datos de ${ }^{14} \mathrm{C}$ (antes y después de su calibración) les provean más que el siglo correcto, en el mejor de los casos.” (La traducción es mía).

Sin embargo, el potencial de la presente propuesta para la construcción de cronologías regionales e interregionales se basa en el hecho que, desde hace décadas, se sabe que existe cierta relación entre la variación de la actividad solar y los cambios climáticos a nivel global (p.e. Anderson 1992; Beer 2005; Bray 1971; Haigh 2007; Lohmann, Rimbu \& Dima 2004; Suess 1967, 1968; Svensmark \& FriisChristensen 1997).

La relación entre clima y Arqueología fue explorada primero por el arqueólogo alemán Friedrich E. Zeuner, del Museo Británico de Historia Natural de Londres, Inglaterra, quien en 1946 publicó su libro Dating the Past: An Introduction to Geochronology, donde desarrolló una variedad de tópicos geofísicos, muchos de ellos dependientes del clima y relacionados a la irradiancia solar total (Renfrew 1990: 657). 
No obstante, tras la euforia inicial de los estudios sobre Ecología Humana de la década de 1950, la comunidad arqueológica ha sido más bien escéptica acerca de evocar fenómenos climáticos para entender aspectos del registro arqueológico que no podían ser explicados por otros medios (Kuniholm 1990: 645). Incluso, el escepticismo del arqueólogo estadounidense P. Kuniholm (1990: 645), lo llevó al extremo de plantear serias dudas acerca de la existencia del cambio climático presente y pasado. Veinte años después, el cambio climático nos parece una hecho difícil de cuestionar, dada la gran cantidad de evidencia positiva disponible.

Eso no quiere decir que los geofísicos y demás científicos involucrados en el estudio del ${ }^{14} \mathrm{C}$ y las variaciones climáticas, estén exentos de errores. Basta citar el hecho de que Libby, al notar las discrepancias entre su modelo de Reservorio de Intercambio de Carbono (que asumía un ratio de decaimiento constante del ${ }^{14} \mathrm{C}$ ) y los fechados obtenidos de tumbas egipcias de 'fecha conocida' por medios históricos, llegó a sugerir que era la Egiptología (y no la Geofísica) la que se había equivocado (Renfrew 1990: 657). Los trabajos en series dendrocronológicas, junto con los esfuerzos de Suess, Stuiver y otros, llevaron al desarrollo de las curvas de calibración y la reivindicación de la Egiptología y, por ende, demostraron que Libby se había equivocado, aunque nadie le resta el mérito por inventar el método de datación por radiocarbono, pues sin ello ni siquiera se trataría el tema en estas páginas.

El antropólogo y geofísico australiano nacionalizado estadounidense Rainer Berger, sugirió que los 'wiggles' de Suess podían sustentarse a partir de datos históricos y arqueológicos (Berger 1985). Otros autores intentaron correlacionar eventos arqueológicos e históricos con los grandes cambios climáticos ocurridos entre fines del Pleistoceno y durante el Holoceno (Eddy 1977: 88; Perry \& Hsu 2000; Salcedo 2006).

Sin embargo, no es mi intención caer en la "ciclomanía" advertida por el astrónomo inglés Sir Joseph N. Lockyer (1874 [citado en Hoyt \& Schatten 1997: 165]). Al respecto, Hoyt \& Schatten (1997: 165-166) escribieron lo siguiente:

\begin{abstract}
"[...] buscar ciclos es ciertamente un prospecto atractivo. [...] Desde un punto de vista práctico, un ciclo puede ser considerado importante solo si puede ser ploteado. Si sofisticados análisis son requeridos para detectar el ciclo, este quizás tiene solo una importancia secundaria. Aunque estos criterios no son los criterios matemáticos usuales para [determinar la] significancia [estadística], son una guía práctica y con los pies en la tierra para lo que es importante. Juzgado por esos criterios, el ciclo de manchas solares es altamente significativo porque es fácilmente visto en los datos. [...] Cuando se juzga la utilidad de cualquier ciclo prominente, la pregunta crucial es si existe un mecanismo físico para explicarlo. Si no, el ciclo puede surgir por mero azar. Más aún, nuevas ideas emergentes en la teoría del caos requieren el reconocimiento de fenómenos 'cíclicos' en sistemas caóticos, como las turbulentas atmósferas terrestre y solar, que pueden no exhibir periodicidades estrictas." (La traducción es mía).
\end{abstract}

Siendo sarcástico, puedo decir que es mi anhelo que en un futuro cercano otros investigadores prueben y corroboren la validez del presente modelo.

\title{
Agradecimientos
}

Quiero agradecer especialmente a Pieter Van Dalen Luna, director del Museo de Arqueología y Antropología de San Marcos, por invitarme a participar de la presente publicación. Las siguientes bibliotecas me brindaron su apoyo: Biblioteca Central, Hemeroteca de Ciencias y Servicio de Provisión de Documentos de la Pontificia Universidad Católica del Perú (PUCP); y Biblioteca del Instituto Francés de Estudios Andinos (IFEA). Asimismo, agradezco a mis colegas c.Dr. Jonathan Dubois y Mag. Emily Baca, por facilitarme varios artículos de difícil acceso. 


\section{BiBLIOGRAFÍA}

ANDERSON, E. C., W. F. LIBBY, S. WEINHOUSE, A. F. REID, A. D. KIRSHENBAUM y A. V. GROSSE

1947 "Radiocarbon from Cosmic Radiation". Science 105 (2735): 576-577.

Anderson, R. Y.

1992 "Possible Connection between Surface Winds, Solar Activity and the Earth's Magnetic Field". Nature 358 (6381): 51-53.

ARNOLD, J. R. y E. C. ANDERSON

1957 "The Distribution of Carbon-14 in Nature". Tellus 9 (1): 28-32.

ARNOLD, J. R. y W. F. LIBBY

1949 “Age Determinations by Radiocarbon Content: Checks with Samples of Known Age". Science 110 (2869): 678-680.

BEER, J.

2005 "Solar Variability and Climate Change". Global Change Newsletter 63: 18-20. Berger, C. R. A.

1983 "New Dating Techniques". En: Early Man in the New World, R. Shutler Jr. (ed.). Beverly Hills, CA: SAGE, pp. 159-161.

1985 “Suess 'Wiggles and Deviations' Proven by Historical and Archaeological Means". Meteoritics 20 (2): 395-401.

BOWMAN, S.

1990 Radiocarbon Dating. Berkeley: University of California Press / London: British Museum, Interpreting the Past, 64p.

BLACKWELL, P. G. y C. E. BUCK

2003 “Estimating Radiocarbon Calibration Curves". Bayesian Analysis 1 (1): 1-21.

BRAY, J. R.

1967 "Variation in Atmospheric Carbon-14 Activity Relative to a Sunspot -Auroral Solar Index". Science 156 (3775): 640-642.

1971 "Solar-Climate Relationships in the Post-Pleistocene”. Science 171 (3977): 1242-1243.

BROECKER, W. S., E. A. OLSON y J. B. BIRD

1959 "Radiocarbon Measurements on Samples of Known Age". Nature 183 (4675): 1582-1584.

BRONK-RAMSEY, C.

2005 OxCal Calibration Program, v3.10r. Oxford Radiocarbon Accelerator Unit, Research Laboratory for Archaeology and the History of Art, Oxford University, Oxford.

BRONK-RAMSEY, C., C. E. BUCK, S. W. MANNING, P. REIMER y H. VAN DER PLICHT

2006 "Developments in Radiocarbon Calibration for Archaeology". Antiquity 80 (310): 783-798.

BUCHA, V.

1969 “Changes of the Earth's Magnetic Moment and Radiocarbon Dating”. Nature 224 (5220) 681-683.

CHARVÁTOVÁ, I.

2000 "Can Origin of the 2400-Year Cycle of Solar Activity be Caused by Solar Inertial Motion?" Annales Geophysicae 18: 399-405.

CLARK, R. M.

1975 “A Calibration Curve for Radiocarbon Dates”. Antiquity 49 (196): 251-266.

1979 "Calibration, Cross-Validation and Carbon-14, I". Journal of the Royal Statistical Society A 142 (1): 4762.

CROWE, C.

1958 "Carbon-14 Activity during the Past 5,000 Years". Nature 182 (4633): 470-471.

DAMON, P.E. y T. W. LINICK

1986 "Geomagnetic-Heliomagnetic Modulation of Atmospheric Radiocarbon Production". Radiocarbon 28 (2A): 266-278.

1962 “Arizona Radiocarbon Dates III”. Radiocarbon 4 (1): 239-249. 
DAMON, P. E. y R. E. STERNBERG

1989 “Global Production and Decay of Radiocarbon". Radiocarbon 31 (3): 697-703.

DAMON, P. E., S. CHENG \& T. W. LINICK

1989 "Fine and Hyperfine Structure in the Spectrum of Secular Variations in Atmospheric 14C". Radiocarbon 31 (3): 704-718.

DAMON, P. E., J. C. LERMAN y A. LONG

1978 "Temporal Fluctuations of Atmospheric 14C: Causal Factors and Implications". Annual Review of Earth and Planetary Sciences 6: 457-494.

DE JONG, A. F. M., W. G. MOOK y B. BECKER

1979 “Confirmation of the Suess Wiggles: 3200-3700 BC". Nature 280 (5717): 48-49.

DE VRIES, $\mathrm{H}$.

1958 "Variation in Concentration of Radiocarbon with Time and Location on Earth". Proceedings of the Koninklijke Nederlandse Akademie van Wetenschappen B 61: 94-102.

1959 "Measurement and Use of Natural Radiocarbon". En: Researches in Geochemistry, P. H. Abelson (ed.). New York: John Wiley \& Sons, pp. 169-189.

DERGACHEV, V. A., O. M. RASPOPOV y S. S. VASILIEV

2000 "Long-Term Variability of Solar Activity during the Holocene". En: Proceedings of the 1st Solar and Space Weather Euroconference "The Solar Cycle and Terrestrial Climate», Santa Cruz de Tenerife, 25-29 Set.2000, A. Wilson (ed.). Noordwijk: ESA, SP-463, pp. 489-492.

DEWEY, E. R.

1960 "The 200-Year Cycle in the Length of the Sunspot Cycle". Journal of Cycle Research 9 (2): 67-82.

DOUGLASS, A. E.

1943 “Advances in Dendrochronology, 1943". Tree-Ring Bulletin 9 (3): 18-24.

EDDY, J. A.

1976 “The Maunder Minimum”. Science 192 (4245): 1189-1202.

1977 "The Case of the Missing Sunspots". Scientific American 236 (5): 80-88, 92.

1980 "Climate and the Role of the Sun". Journal of Interdisciplinary History 10 (4): 725-747.

FRICK, P., D. GALYAGIN, D.V. HOYT, E. NESME-RIBES, K.H. SCHATTEN, D. SOKOLOFF y V. ZAKHAROV

1997 "Wavelet Analysis of Solar Activity Recorded by Sunspot Groups". Astronomy and Astrophysics 328: 670-681.

GLEISSBERG, W.

1939 “A Long-Periodic Fluctuation of the Sun-spot Numbers". The Observatory 62: 158-159.

1971 "The Probable Behaviour of Sunspot Cycle 21". Solar Physics 21 (1): 240-245.

HAIGH, J. D.

2007 "The Sun and the Earth's Climate". Living Review of Solar Physics 4 (2), 64p.

HALE, G. E.

1908 "On the Probable Existence of a Magnetic Field in Sun-Spots". The Astrophysical Journal 28: 315-343 + Pl. 26-29.

HOUTERMANS, J. C.

1966 "On the Quantitative Relationships between Geophysical Parameters and the Natural C14 Inventory". Zeitschrift für Physik A 193 (1): 1-12.

HOYT, D. V. y K. H. SCHATTEN

1997 The Role of the Sun in Climate Change. Oxford: Oxford University Press, 279p.

HUGHEN, K. A., S. LEHMAN, J. R. SOUTHON, J. OVERPECK, O. MARCHAL, C. HERRING y J. TURNBULL

2004 1"4C Activity and Global Carbon Cycle Changes over the Past 50,000 Years". Science 303 (5655): 202207.

KROMER, B.

2009 "Radiocarbon and Dendrochronology". Dendrochronologia 27 (1): 15-19. 
KROMER, B. y B. BECKER

1993 “German Oak and Pine 14C Calibration, 7200-9400 BC". Radiocarbon 35 (1): 125-135.

KUNIHOLM, P. I.

1990 "Archaeological Evidence and Non-Evidence for Climatic Change". Philosophical Transactions of the Royal Society of London A 330 (1615): 645-655.

LEÓN, E.

2006 "Radiocarbono y Calibración: Potencialidades para la Arqueología Andina". Arqueología y Sociedad 17: 67-89.

LIBBY, W. F.

1946 “Atmospheric Helium Three and Radiocarbon from Cosmic Radiation". Physical Review 69 (11-12): 671-672.

1960 Radiocarbon Dating. Nobel Lecture, December 12, pp. 593-610.

1963 "Accuracy of Radiocarbon Dates". Science 140 (3564): 278-280.

1967 "History of Radiocarbon Dating". En: Radioactive Dating and Methods of Low-Level Counting: Proceedings of Symposium Organized by the International Atomic Energy Agency (IAEA) and the Joint Commission on Applied Radioactivity, Monaco, 02-10 Mar.1967. Vienna: IAEA, pp. 3-25.

1973 "Radiocarbon Dating, Memories and Hopes". En: Proceedings of the 8th International Conference on Radiocarbon Dating, Royal Society of New Zealand, Wellington, 1972, pp. 27-43.

1980 “Archaeology and Radiocarbon Dating". Radiocarbon 22 (4): 1017-1020.

LIBBY, W. F., E. C. ANDERSON y J. R. ARNOLD

1949 “Age Determination by Radiocarbon Content: World-Wide Assay of Natural Radiocarbon”. Science 109 (2827): 227-228.

LINK, F.

1955 "Variations Séculaires des Climats et de l'Activité Solaire dans les Quatre Derniers Millénaires". Bulletin of the Astronomical Institute of Czechoslovakia 6: 122.

1961 "La Rotation Terrestre et l'Activité Solaire". Bulletin of the Astronomical Institute of Czechoslovakia 12 (1): 70-71.

1963 "Variations á Longues Périodes de l'Activité Solaire avant le 17ème Siècle". Bulletin of the Astronomical Institute of Czechoslovakia 14 (6): 226.

1967 "Relations entre les Variations Climatiques et la Rotation Terrestre". Bulletin of the Astronomical Institute of Czechoslovakia 18 (1): 48.

LOHMANN, G., N. RIMBU y M. DIMA

2004 "Climate Signature of Solar Irradiance Variations: Analysis of Long-Term Instrumental, Historical, and Proxy Data". International Journal of Climatology 24: 1045-1056.

MCELHINNY, M. W. y W. E. SENANAYAKE

1982 "Variations in the Geomagnetic Dipole, 1: The Past 50,000 Years". Journal of Geomagnetism and Geoelectricity 34 (1): 39-51.

MÖRNER, N.-A. y D. RICKARD

1974 "Quantitative Analyses of Eustatic Changes and Climatic Cycles". En: Les Méthodes Quantitatives d'Étude des Variations du Climat au Cours du Pléistocène: Actes du Colloque International à Gif-surYvette, 05-09 Jun.1973, J. Labeyrie (ed.). Paris: Centre National de la Recherche Scientifique (CNRS), Colloques Internationaux du CNRS 219, pp. 143-153.

MÜNNICH, K. O.

1957 “Heidelberg Natural Radiocarbon Measurement I". Science 126 (3266): 194-199.

MUSCHELER, R., J. BEER, P. W. KUBIK y H.-A. SYNAL

2005 "Geomagnetic Field Intensity during the Last 60,000 Years Based on 10Be and 36Cl from the Summit Ice Cores and 14C”. Quaternary Science Reviews 24 (16-17): 1849-1860. 
NEFTEL, A., H. OESCHGER y H. E. SUESS

1981 "Secular Non-Random Variations of Cosmogenic Carbon-14 in the Terrestrial Atmosphere". Earth and Planetary Science Letters 56: 127-147.

PAZDUR, A. y M. F. PAZDUR

1994 "Fundamental Concepts and Archaeological Applications of the Radiocarbon Dating Method". En: Andes: Radiocarbon Database for Bolivia, Ecuador and Peru, M. S. Ziółkowski, M. F. Pazdur, A. Krzanowski \& A. Michczyński (eds.). Warszawa: Andean Archaeological Mission of the Institute of Archaeology, Warsaw University / Gliwice: Gliwice Radiocarbon Laboratory of the Institute of Physics, Silesian Technical University, pp. 25-62.

PERRY, C. A. y K. J. HSU

2000 "Geophysical, Archaeological, and Historical Evidence Supports a Solar Model for Climate Change". Proceedings of the National Academy of Sciences 97 (23): 12433- 12438.

RALPH, E. K.

1959 “University of Pennsylvania Radiocarbon Dates III". Radiocarbon 1 (1): 45-48.

RALPH, E. K. y R. STUCKENRATH

1960 “Carbon-14 Measurements of Known Age Samples". Nature 188 (4746): 185-187.

REIMER, P. J., M. G. L. BAILLIE, E. BARD, A. BAYLISS, J. WARREN-BECK, C. J. H. BERTRAND, P. G. BLACKWELL, C. E. BUCK, G. S. BURR, K. B. CUTLER, P. E. DAMON, R. L. EDWARDS, R. G. FAIRBANKS, M. FRIEDRICH, T. P GUILDERSON, A. G. HOGG, K. A. HUGHEN, B. KROMER, G. MCCORMAC, S. W. MANNING, C. BRONK-RAMSEY, R. W. REIMER, S. REMMELE, J. R. SOUTHON, M. STUIVER, S. TALAMO, F. W. TAYLOR, J. VAN DER PLICHT y C. E. WEYHENMEYER

2004 "IntCal04 Terrestrial Radiocarbon Age Calibration, 0-26 Cal kyr BP". Radiocarbon 46 (3): 10291058.

REIMER, P. J., M. G. L. BAILLIE, E. BARD, A. BAYLISS, J. WARREN-BECK, P. G. BLACKWELL, C. BRONK-RAMSEY, C. E. BUCK, G. S. BURR, R. L. EDWARDS, M. FRIEDRICH, P. M. GROOTES, T. P GUILDERSON, I. HAJDAS, T. J. HEATON, A. G. HOGG, K. A. HUGHEN, K. F. KAISER, B. KROMER, G. MCCORMAC, S. W. MANNING, R. W. REIMER, D. A. RICHARDS, J. R. SOUTHON, S. TALAMO, C. S. M. TURNEY, J. VAN DER PLICHT y C. E. WEYHENMEYER

2009 "IntCal09 and Marine09 Radiocarbon Age Calibration Curves, 0-50,000 Years Cal BP". Radiocarbon 51 (4): 1111-1150.

RENFREW, A. C.

1990 "Climate and Holocene Culture Change: Some Practical Problems". Philosophical Transactions of the Royal Society of London A 330 (1615): 657-663.

RUNCORN, S. K.

1990 “Closing Remarks: Geophysical”. Philosophical Transactions of the Royal Society of London A 330 (1615): 685-687.

SALCEDO, L. E.

1997 Excavaciones en Cerro Tres Marías (Valle de Lurín): Un Campamento del Periodo Arcaico en las Lomas de Atocongo. Tesis de Licenciatura. Lima: Pontificia Universidad Católica del Perú, 448p. URL: http:// tesis.pucp.edu.pe/repositorio/handle/123456789/749?show=full.

1998 "Una Aproximación al Periodo Arcaico del Área Central Andina”. Andesita 1 (2): 1-42.

2001 “El Complejo Lauricocha de los Andes Centrales". En: XII Congreso Peruano delHombre y la Cultura Andina «Luis G. Lumbreras», Ayacucho, 24-29 Oct.1999, I. Pérez, W. Aguilar \& M. Purizaga (eds.). Ayacucho: Universidad Nacional San Cristóbal de Huamanga, vol. 2, pp. 295-312.

2006 Prehistoria Sudamericana: Modelo de Migraciones Múltiples para el Doblamiento Temprano y Evolución Cultural de Sudamérica. Tesis de Doctorado. Varsovia: Universidad de Varsovia, 860p.

2007 La Calibración Radiocarbónica y el Establecimiento de una División del Tiempo a partir de las Variaciones Seculares del Carbono-14 en la Atmósfera. Ponencia presentada en el IV Coloquio de Estudiantes de Arqueología PUCP. Lima: Pontificia Universidad Católica del Perú, 14 de Abril de 2007. 
2011a Tempus Solaris: Algunas nociones sobre la actividad solar, las variaciones seculares del Carbono-14 atmosférico y los Estadios del Isótopo de Carbono. Lima: Intersur Concesiones S.A. - Servicios Gráficos Rodríguez Paredes, XVIIIp. y 190p. URL: https://sites.google.com/site/tempussolaris/. Disponible en LIBUN: www.libun.udu.pe, ISBN 978-612-00-0581-1.

2011b Datación por Radiocarbono: Un gran aporte de la Química a la Arqueología. Ponencia presentada en el Encuentro PUCP por el Año Internacional de Química 2011, Lima, del 22 al 24 de Agosto de 2011.

SCHOVE, D. J.

1983 "Sunspot, Auroral, Radiocarbon and Climatic Fluctuations since 7000 BC". Annales Geophysicae 1 (4-5): 391-396.

SONETT, C. P.

1984 "Very Long Solar Periods and the Radiocarbon Record". Reviews of Geophysics and Space Physics 22 (3): 239-254.

1985 “Suess ‘Wiggles’: A Comparison between Radiocarbon Records”. Meteoritics 20 (2): 383-393.

SONETT, C. P. y H. E. SUESS

1984 "Correlation of Bristlecone Pine Ring Widths with Atmospheric 14C Variations: A Climate-Sun Relation". Nature 307 (5947): 141-143.

STERNBERG, R. S. y P. E. DAMON

1983 “Atmospheric Radiocarbon: Implications for the Geomagnetic Dipole Moment". Radiocarbon 25 (2): 239-248.

STUIVER, M.

1961 "Variations in Radiocarbon Concentration and Sunspot Activity". Journal of Geophysical Research 66 (1): 273-276.

1965 "Carbon-14 Content of 18th-and 19th-Century Wood Variations Correlated with Sunspot Activity". Science 149 (3683): 533-534.

1970 “Tree Ring, Varve and Carbon-14 Chronologies”. Nature 228 (5270): 454-455.

1980 "Solar Variability and Climatic Change during the Current Millennium". Nature 286 (5776): 868871.

STUIVER, M. y B. BECKER

1993 "High-Precision Decadal Calibration of the Radiocarbon Time Scale, AD 1950-6000 BC". Radiocarbon 35 (1): 35-65.

STUIVER, M. y T. F. BRAZIUNAS

1989 "Atmospheric 14C and Century-Scale Solar Oscillations". Nature 338 (6214): 405-408.

STUIVER, M. y G. W. PEARSON

1993 "High-Precision Bidecadal Calibration of the Radiocarbon Time Scale, AD 1950-500 BC and 25006000 BC". Radiocarbon 35 (1): 1-23.

STUIVER, M. y H. A. POLACH

1977 “Reporting of 14C Data". Radiocarbon 19 (3): 355-363.

STUIVER, M. y P. D. QUAY

1980 "Changes in Atmospheric Carbon-14 Attributed to a Variable Sun". Science 207 (4426): 11-19.

STUIVER, M. y H. E. SUESS

1966 "On the Relationship between Radiocarbon Dates and True Ages". Radiocarbon 8 (1): 534-540.

STUIVER, M., P. J. REIMER, E. BARD, J. W. BECK, G. S. BURR, K. A. HUGHEN, B. KROMER, G. MCCORMAC, J. VAN DER PLICHT y M. SPURK

1998 “IntCal98 Radiocarbon Age Calibration 24,000-0 Cal BP”. Radiocarbon 40 (3): 1041-1083.

SUESS, H. E.

1961 "Secular Changes in the Concentration of Atmospheric Radiocarbon". En: Proceedings of the Conference on Problems Related to Interplanetary Matter, Highland Park, IL, 20-22 Jun.1960. Washington, D.C.: NAS-NRC Publication 845, National Science Report 33, pp. 90-95. Interpretations". Journal of Geophysical Research 70 (23): 5937-5952. 
1967 "Bristlecone-Pine Calibration of the Radiocarbon Time-Scale from 4100 B.C. to 150 B.C". En: Radioactive Dating and Methods of Low-Level Counting: Proceedings of Symposium organized by the International Atomic Energy Agency (IAEA) and the Joint Commission on Applied Radioactivity, Monaco, 02-10 Mar.1967. Vienna: IAEA, pp. 143-151.

1968 "Climatic Changes, Solar Activity, and the Cosmic-Ray Production Rate of Natural Radiocarbon". Meteorological Monographs 8 (30): 146-150.

1970a "Bristlecone-Pine Calibration of the Radiocarbon Time-Scale: 5200 B.C. to the Present". En: Radiocarbon Variations and Absolute Chronology: Proceedings of the 12th Nobel Symposium, Institute of Physics at Uppsala University, Uppsala, 11-15 Ago.1969, I. U. Olsson (ed.). Stockholm: Almquist \& Wiksell-Gebers Forlag AB, pp. 303-311.

1970b "The Three Causes of the Secular Carbon-14 Fluctuations, their Amplitudes and Time Constants". En: Radiocarbon Variations and Absolute Chronology: Proceedings of the 12th Nobel Symposium, Institute of Physics at Uppsala University, Uppsala, 11- 15 Ago.1969, I. U. Olsson (ed.). Stockholm: Almquist \& Wiksell-Gebers Forlag AB, pp. 595-605.

1974 "Natural Radiocarbon Evidence Bearing on Climatic Changes". En: Les Méthodes Quantitatives d'Étude des Variations du Climat au Cours du Pléistocène: Actes du Colloque International à Gif-sur-Yvette, 05-09 Jun.1973, J. Labeyrie (ed.). Paris: Centre National de la Recherche Scientifique (CNRS), Colloques Internationaux du CNRS 219, pp. 311-317.

1978 "La Jolla Measurements of Radiocarbon in Tree-Ring Dated Wood". Radiocarbon (1): 1-18.

1980 "The Radiocarbon Record in Tree Rings of the Last 8000 Years". Radiocarbon 22 (2): 200-209.

1986 "Secular Variations of Cosmogenic 14C on Earth: Their Discovery and Interpretation". Radiocarbon 28 (2A): 259-265.

SUESS, H. E. y T. W. LINICK

1990 The 14C Record in Bristlecone Pine Wood of the Past 8000 Years Based on the Dendrochronology of the Late C. W. Ferguson. Philosophical Transactions of the Royal Society of London A 330 (1615): 403412.

SVENSMARK, H. y E. FRIIS-CHRISTENSEN

1997 "Variation of Cosmic Ray Flux and Global Cloud Coverage-A Missing Link in Solar-Climate Relationships". Journal of Atmospheric and Solar-Terrestrial Physics 59 (11): 1225-1232.

TAYLOR, R. E.

1985 "The Beginnings of Radiocarbon Dating in American Antiquity: A Historical Perspective". American Antiquity 50 (2): 309-325.

USOSKIN, I. G.

2008 “A History of Solar Activity over Millennia". Living Review of Solar Physics 5 (3), 88p.

USOSKIN, I. G., D. SOKOLOFF y D. MOSS

2009 "Grand Minima of Solar Activity and the Mean-Field Dynamo". Solar Physics 254 (2): 345-355.

USOSKIN, I. G., S. K. SOLANKI y G. A. KOVALTSOV

2007 "Grand Minima and Maxima of Solar Activity: New Observational Constraints". Astronomy and Astrophysics 471: 301-309.

VELARDE, L.

1998 "Problemática de los Fechados Carbono 14 y su Calibración: El Caso del Formativo de los Andes Centrales". Arqueología y Sociedad 12: 11-28.

VOGEL, J. C.

2002 "Secular Variations in Carbon-14 and their Geophysical Implications". South African Journal of Science 98 (2): 154-160.

WEISS, N. O.

1990 "Periodicity and Aperiodicity in Solar Magnetic Activity." Philosophical Transactions of the Royal Society of London A 330 (1615): 617-625.

WENDLAND, W. M. y D. L. DONLEY

1971 “Radiocarbon-Calendar Age Relationship". Earth and Planetary Science Letters 11: 135-139. 
WIGLEY, T. M. L. y P. M. KELLY

1990 "Holocene Climatic Change, 14C Wiggles and Variations in Solar Irradiance". Philosophical Transactions of the Royal Society of London A 330 (1615): 547-560.

WILLIS, E. H., H. TAUBER y K. O. MÜNNICH

1960 "Variations in the Atmospheric Radiocarbon Concentration over the Past 1300 Years". Radiocarbon 2 (1): $1-4$.

ZIÓŁKOWSKI, M. S., M. F. PAZDUR, A. KRZANOWSKI y A. MICHCZYŃSKI (eds.)

1994 Andes: Radiocarbon Database for Bolivia, Ecuador and Peru. Warszawa: Andean Archaeological Mission of the Institute of Archaeology, Warsaw University / Gliwice: Gliwice Radiocarbon Laboratory of the Institute of Physics, Silesian Technical University. 